\title{
COMPLETENESS THEOREMS, INCOMPLETENESS THEOREMS AND MODELS OF ARITHMETIC ${ }^{(1)}$
}

BY

\author{
KENNETH MC ALOON
}

\begin{abstract}
Let $\mathbb{Q}$ be a consistent extension of Peano arithmetic and let $\mathbb{Q}_{n}^{0}$ denote the set of $\Pi_{n}^{0}$ consequences of $\mathcal{Q}$. Employing incompleteness theorems to generate independent formulas and completeness theorems to construct models, we build nonstandard models of $\mathbb{Q}_{n+2}^{0}$ in which the standard integers are $\Delta_{n+1}^{0}$-definable. We thus pinpoint induction axioms which are not provable in $\mathscr{Q}_{n+2}^{0}$; in particular, we show that (parameter free) $\Delta_{1}^{0}$-induction is not provable in Primitive Recursive Arithmetic. Also, we give a solution of a problem of Gaifman on the existence of roots of diophantine equations in end extensions and answer questions about existentially complete models of $\mathscr{C}_{2}^{0}$. Furthermore, it is shown that the proof of the Gödel Completeness Theorem cannot be formalized in $\mathbb{C}_{2}^{0}$ and that the MacDowell-Specker Theorem fails for all truncated theories $\mathbb{E}_{n}^{0}$.
\end{abstract}

The completeness theorems of the title are the usual completeness and compactness theorems for the predicate calculus and strong variants of them which are obtained by virtue of the fact that the Henkin proof of the usual completeness theorem can be formalized in arithmetic. The incompleteness theorems are the Gödel theorems and the variants due to Rosser and Mostowski.

Using model theoretic methods, Ryll-Nardzewski and also Rabin proved that (Peano) arithmetic was not finitely axiomatizable by showing that all finite subsystems of arithmetic have nonstandard models in which some induction axiom fails. On the other hand, using proof-theoretic methods, Mostowski and also Montague showed that in arithmetic one can prove the consistency of any finite subsystem of arithmetic, which again establishes nonfinite axiomatizability. In this paper we combine these techniques playing off the completeness theorems against the incompleteness theorems and construct nonstandard models of subsystems of arithmetic in which the standard integers and definable ( $\$ \S 3$ and 6 ). The nonfinite axiomatizability results that we obtain in this way are optimal (from the point of view of quantifier complexity). We introduce the notion of representability of a theory in a model, cf. $\$ 2$, which enables us to apply the incompleteness

Received by the editors August 4, 1976.

AMS (MOS) subject classifications (1970). Primary 02H05, 02H25, $02 \mathrm{G} 15$.

(')Various results of this paper were announced in [Mc].

() American Mathematical Society 1978 
theorems to non(recursively) axiomatizable theories. Our methods thus work equally well for axiomatizable and nonaxiomatizable extensions of Peano arithmetic. We also obtain other results of "foundational interest": in $\$ 4$ we comment on Feferman's work on consistency statements, in $\$ 6$, we show that (parameter free) $\Delta_{1}^{0}$-induction is not provable in Primitive Recursive Arithmetic (Theorem 6.8), and in \$7, we show that the proofs of the Gödel and Henkin completeness theorems cannot be formalized in weak subsystems of arithmetic such as that axiomatized by the true $\Sigma_{3}^{0}$ sentences, Theorem 7.5.

Along the way we also prove some theorems of "model-theoretic interest." For example, in $\$ 3$ we give a solution of a problem of Gaifman on roots of diophantine equations in end extensions, Corollary 2.7; in \$4, we show that recursively enumerable extensions of Peano arithmetic have models with arbitrarily small existentially and universally definable nonstandard points, Theorem 4.8. In §5, we apply our techniques to answer some questions about existentially complete structures; moreover, in \$7, we show that the MacDowell-Specker Theorem does not hold for various (strong) subsystems of arithmetic, Theorem 7.2.

We would like to thank our colleagues M. Dickman, J. Y. Girard, J. L. Krivine and G. Sabbagh of the Équipe de Logique of the University of Paris VII for many interesting discussions on the subject matter of this paper. Also, we would like to thank the Logic Group at The Rockefeller University for their hospitality while this paper was being written.

1. Preliminaries. Let $\mathcal{L}$ be a first order language with equality whose nonlogical constants are symbols $+, \cdot, 0,1$. In this paper we shall deal with (consistent) theories formulated in $\mathcal{E}$ such as first order Peano arithmetic. We let $\mathcal{G}$ denote "true arithmetic," meaning the set of sentences satisfied in the standard integers $\mathbf{N}$. We let $\mathscr{P}$ stand for Peano arithmetic and $\mathcal{Q}$ will always denote a consistent extension of $\mathscr{P}$; we denote by $\mathbb{Q}_{m}^{0}$ the set of $\Pi_{m}^{0}$ consequences of $\mathcal{Q}$. We shall suppose that syntactic notions such as formula, variable etc. have been defined in arithmetic terms, as in [Fef]; we thus are identifying formulas etc. with their Gödel numbers.

Let $\Re$ be a structure for $\mathcal{L}$ and let $F\left(v_{1}, \ldots, v_{s}\right)$ be a formula of $\mathcal{L}$. For $k_{1}, \ldots, k_{s} \in|\Re|$, we write $\Re \vDash F\left[k_{1}, \ldots, k_{s}\right]$ iff the assignment $\left\langle k_{1}, \ldots, k_{s}\right\rangle$ satisfies $F\left(v_{1}, \ldots, v_{s}\right)$ in $\mathfrak{R}$. By $\mathcal{L}(\mathfrak{T})$ we shall mean the

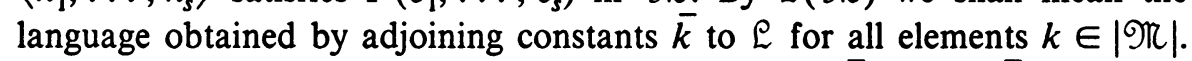
So we have the trivial equivalence $\Re \vDash F\left(\bar{k}_{1}, \ldots, \bar{k}_{s}\right) \Leftrightarrow \mathscr{N} \vDash$ $F\left[k_{1}, \ldots, k_{s}\right]$. If $\Re$ is a substructure of $\Re$, we say that $\Re$ is a $\forall^{n}$-elementary subsystem of $\Re$ iff

$$
\Re \vDash F\left[k_{1}, \ldots, k_{s}\right] \Leftrightarrow \Re \vDash F\left[k_{1}, \ldots, k_{s}\right]
$$

for all $\forall^{n}$-formulas $F$ and for all $k_{1}, \ldots, k_{s} \in|\mathscr{T l}|$; in this case we write $\Re<_{n} \Re$. 
Following Gaifman [G], we note that by Matiasevich's work, every $\Pi_{n}^{0}$-formula is equivalence in $\mathscr{P}$ to a $\forall^{n}$-formula; therefore, when $\Re$ and $\mathscr{\Re}$ are models of $\mathscr{P}_{n+2}^{0}$ and $\mathscr{N}$ is a $\forall^{n}$-elementary substructure of $\mathscr{N}, \mathfrak{R}$ is in fact a $\Pi_{n}^{0}$-elementary substructure of $\mathfrak{\Re}$. Thus we have

THEOREM 1.1. Let $\mathfrak{R}$ and $\Re$ be models of $\mathscr{P}_{n+2}^{0}$ such that $\Re$ is a $\forall^{n}$-elementary substructure of $\Re$. For all $\Pi_{n}^{0}$-formulas $F\left(v_{1}, \ldots, v_{s}\right)$ and all $k_{1}, \ldots, k_{s} \in|\Re|$, we have

$$
\Re \vDash F\left[k_{1}, \ldots, k_{s}\right] \Leftrightarrow \Re \vDash F\left[k_{1}, \ldots, k_{s}\right] .
$$

Let $\operatorname{Prf}\left(u ; v_{1}, \ldots, v_{s} ; w\right)$ be a $\Pi_{0}^{0}$ formula which expresses in $\mathcal{P}$ that " $u$ is a proof in the predicate calculus of $w$ from the hypotheses $v_{1}, \ldots, v_{s}$ "; cf. [Fef] or [Sch]. Let us set $\operatorname{Prov}\left(v_{1}, \ldots, v_{s} ; w\right)$ to be the formula

$$
\exists u \operatorname{Prf}\left(u ; v_{1}, \ldots, v_{s} ; w\right) \text {; }
$$

the formula $\operatorname{Prov}\left(v_{1}, \ldots, v_{s} ; w\right)$ then expresses in $\mathscr{P}$ that " $w$ is provable in the predicate calculus from $v_{1}, \ldots, v_{s}$ ".

We denote by $\bar{n}$ the term $0+1+\cdots+1$ ( $n$-times) of $\mathcal{L}$ which denotes the natural number $n$. This bar function "-" is a map from $\mathbf{N}$ to terms of $\mathcal{L}$. The function defined in $\mathcal{P}$ which represents the bar function will be denoted $y=\tilde{x}$; that is, " $"$ " denotes the formalized version of " $-"$.

In [Mon], also cf. [K, L] and [Sm], it is shown how to construct, for $n \geqslant 0$, a formula $\operatorname{Tr}_{n}(v)$ which is a "truth definition" in $\mathcal{P}$ for the class of $\forall^{n}$ sentences, such that for $n=0$, the truth definition is a $\Delta_{1}^{0}$-predicate and for $n>0$, it is $\Pi_{n}^{0}$. We then have

Scheme 1.2. $\mathscr{P} \vdash F \leftrightarrow \operatorname{Tr}_{n}(\bar{F}), F$ a $\forall^{n}$-sentence.

Moreover the verification of this scheme is elementary and utilizes only a. finite number of facts about $\operatorname{Tr}_{n}(v)$ which can be established in $\mathcal{P}$; in other words, for fixed $n$, there is a finite subsystem $P_{1}, \ldots, P_{s_{n}}$ of $\mathcal{P}$ such that the following scheme holds:

Scheme 1.3. $P_{1} \wedge \cdots \wedge P_{s_{n}} \vdash F \leftrightarrow \operatorname{Tr}_{n}(\bar{F}), F$ a $\forall^{n}$-sentence.

REMARK 1.4. We note for later reference that the proof of Scheme $1.3 \mathrm{can}$ itself be formalized in $\mathscr{P}$.

In the course of the proof of Scheme 1.2 which proceeds by induction on $F$ a somewhat more elaborate result is established, to wit

Scheme 1.5. $\mathscr{P} \vdash \forall v_{1} \cdots \forall v_{s}\left[F\left(v_{1}, \ldots, v_{s}\right) \leftrightarrow \operatorname{Tr}_{n}\left(\bar{F}\left(\tilde{v}_{1}, \ldots, \tilde{v}_{s}\right)\right)\right], F$ a $\forall^{n}$-formula.

Let $\operatorname{Cons}(\phi)$ be the formula $\neg \operatorname{Prov}(\phi ; \bar{\Lambda})$, where $\Lambda$ is the refutable sentence $0 \neq 0$. The formula Cons $(\phi)$, which has free variable $\phi$, asserts in $\mathscr{P}$ that " $\neg \phi$ is not a theorem of the predicate calculus". The following result is also established in [Mon], cf. also [Mos].

THEOREM 1.6. Let $Q$ be a consistent extension of $\mathcal{P}$, let $A_{1}, \ldots, A_{s}$ be 
axioms of $\mathbb{Q}$ and let $n \geqslant 0$. Then $\mathbb{Q} \vdash \forall \phi\left(\operatorname{Tr}_{n}(\phi) \rightarrow \neg \operatorname{Prov}(\phi\right.$, $\left.\overline{A_{1} \wedge \cdots \wedge A_{s}} ; \bar{\Lambda}\right)$ ).

Let $\mathfrak{N}$ be a model of $\mathcal{P}$. We can identify $\mathbf{N}$ with an initial segment of $\Re$; therefore, if $F\left(v_{1}, \ldots, v_{s}\right)$ is a formula of $\mathcal{L}$, since we have supposed syntactic notions to be defined in arithmetic terms, $F\left(v_{1}, \ldots, v_{s}\right)$ is an element of $|\Re|$. If $k_{1}, \ldots, k_{s} \in|\Re|$, we shall denote by $F\left(\bar{k}_{1}, \ldots, \bar{k}_{s}\right)_{\Re}$ the result in $\Re$ of substituting the terms $\tilde{k}_{1}^{\Re}, \ldots, \tilde{k}_{s} \pi$ for $v_{1}, \ldots, v_{s}$ in $F$; in the notation of [Sch], $F\left(\bar{k}_{1}, \ldots, \bar{k}_{s}\right)_{\pi}$ is that element $k$ of $|\Re|$ such that

$\Re \vDash\left(u_{1}=\operatorname{Sub}\left(u_{2}, v_{1}, \ldots, v_{s}, \operatorname{num}\left(u_{3}\right), \ldots, \operatorname{num}\left(u_{s+3}\right)\right)\right)\left[k, F, k_{1}, \ldots, k_{s}\right]$.

Let $\phi_{0} \in|\Re|$ be a (possibly nonstandard) sentence of $\mathcal{L}$ in $\Re$. We define a set $C_{n}^{\phi_{0}}$ of (standard) sentences of $\mathcal{L}(\mathfrak{T})$ as follows:

$F\left(\bar{k}_{1}, \ldots, \bar{k}_{s}\right) \in C_{n}^{\phi_{0}} \Leftrightarrow$ there exists $\psi_{0} \in|\Re|$ such that

$$
\Re \vDash \operatorname{Tr}_{n}\left[\psi_{0}\right] \text { and } \Re \vDash \operatorname{Prov}\left[\psi_{0}, \phi_{0} ; F\left(\bar{k}_{1}, \ldots, \bar{k}_{s}\right)_{\Re}\right] \text {. }
$$

By formalizing the Henkin proof of the Completeness Theorem in $\mathcal{P}, \mathrm{cf} .[\mathbf{H}$, B], [Fef], [Sm] or [B], we have

Theorem 1.7 (The Arithmetized Completeness Theorem). Let $\Re$ be $a$ model of $\mathcal{P}$, let $n \geqslant 0$ and let $\phi_{0} \in|\mathfrak{T}|$. If $\mathscr{\Re} \vDash \forall \phi\left(\operatorname{Tr}_{n}(\phi) \rightarrow \neg \operatorname{Prov}(\phi\right.$, $\left.\bar{\phi}_{0} ; \bar{\Lambda}\right)$ ), then there is a $\forall^{n}$-elementary extension $\Re$ of $\mathscr{N}$ which satisfies $C_{n}^{\phi_{0}}$.

Remarks 1.8. The Arithmetized Completeness Theorem was first formulated by Hilbert and Bernays and later applied by Feferman and by Scott, cf. [H, B], [Fef], [Sco]. More recently it has been applied by Smorynski and by Manewitz, [Sm], [Sm, bis], [Man]. An analogous completeness theorem for set theory and infinitary languages is given by Barwise in [B]. Techniques for working from inside nonstandard models of set theory have been developed by Barwise and Suzuki and Wilmers, cf. [B], [S, W], [Wi]. Our paper [K, M, bis] deals with questions raised by these authors and uses techniques related to those of the present paper. Other techniques for working from inside models of arithmetic are developed by Wilkie in [W].

2. On satisfying formulas in end extensions. Let $\Re$ be a model of $\mathcal{L}$ which is an end extension of the standard integers $\mathbf{N}$. Let $X$ be a subset of $\mathbf{N}$. We say that $X$ is represented in $\Re$ iff there is a formula $R\left(x, y_{1}, \ldots, y_{s}, \bar{b}\right)$ of $\mathcal{L}(\Re)$ such that for all $m \in \mathbf{N}$, we have

$$
m \in X \Leftrightarrow \text { for some } n_{1}, \ldots, n_{s} \in \mathbf{N}, \mathfrak{N} \vDash R\left(\bar{m}, \bar{n}_{1}, \ldots, \bar{n}_{s}, \bar{b}\right) .
$$

If $X$ is a recursively enumerable set, there is a $\Pi_{0}^{0}$-formula $R(u, v)$ which represents $X$ in every model of $\mathcal{P}$; moreover, by Matiasevich's Theorem, cf. [Ma], there is a quantifier free formula $S\left(u, v_{1}, \ldots, v_{s}\right)$ which represents $X$ in 
every model of $\mathscr{P}$. Thus if $Q$ is a recursively enumerable extension of $\mathscr{P}$, then $Q$ is represented by a $\Pi_{0}^{0}$-formula $R(u, v)$ in every model of $Q$; more generally, if $Q$ is $\Sigma_{n+1}^{0}$-definable over $\mathrm{N}$, say by $\exists v_{1} \cdots \exists v_{s} S\left(u, v_{1}, \ldots, v_{s}\right)$ where $S$ is $\Pi_{n}^{0}$, and if $\mathcal{Q}$ is an extension of $\mathscr{T}_{n}^{0}$ (the true $\Pi_{n}^{0}$ sentences), then $\mathcal{Q}$ is represented in all models of $\mathbb{Q}$ by $S\left(u, v_{1}, \ldots, v_{s}\right)$.

Let $E(u, v)$ be a bounded quantifier formula which expresses " $u$ is an element of the finite set coded by $v$ " and which satisfies the following comprehension scheme

$$
\mathscr{P} \vdash \forall x \forall u_{1} \cdots \forall u_{n} \exists v\left[\forall y\left(F\left(y, u_{1}, \ldots, u_{n}\right) \wedge y<x \leftrightarrow E(y, v)\right)\right] .
$$

Let $\mathfrak{R}$ be an end extension of $\mathbf{N}$ and let $Y$ be a subset of $\mathfrak{R}$ which is definable (with parameters) in $\Re$. Then clearly $X=Y \cap \mathrm{N}$ is represented in $\mathfrak{T}$. In this case we say that $X$ is a real number or real of $\mathfrak{T}$. Let us remark that a set can be represented in a model of $\mathcal{P}$ without being a real of the model, cf. [G, K, T]. We also note two further facts. If $Z$ is any set of integers and if $\mathscr{N}$ is a model of $\mathscr{P}$, then there is an elementary extension $\Re$ of $\mathscr{R}$ with an element $c \in|\Re|$ such that $n \in Z \Leftrightarrow \Re \vDash E(\bar{n}, \bar{c})$; this a direct consequence of the compactness theorem. If $\mathfrak{N}$ is a nonstandard model of $\mathcal{P}$ in which the set $Z$ is represented by $S\left(u, v_{1}, \ldots, v_{s}, \bar{b}\right)$ say, then in fact $Z$ is represented in $\mathscr{T}$ by means of a $\Pi_{0}^{0}$-formula $R(u, v, \bar{c})$ with $c \in|\mathscr{T}|$; to see this, let $k$ be an infinite integer of $\mathscr{N}$ and let $c$ code the following "finite" set in $\Re$ :

$$
\left\{\left\langle u, v_{1}, \ldots, v_{s}\right\rangle: u<\bar{k} \wedge v_{1}<\bar{k} \wedge \cdots \wedge v_{s}<\bar{k} \wedge S\left(u, v_{1}, \ldots, v_{s}, \bar{b}\right)\right\} .
$$

Let $\Re$ be a model of $\mathscr{P}$ in which the theory $\mathcal{Q}$ is represented by a $\Pi_{n}^{0}$-formula $R\left(u, v_{1}, \ldots, v_{s}, \bar{b}\right)$. We can define in $\Re$ a function $y=\mathbb{Q}_{x}$ by setting

$$
\begin{aligned}
\mathbb{Q}_{x}=\bigwedge\left\{\phi: \phi \leqslant x \wedge \exists v_{1} \cdots \exists v_{s}\left(v_{1} \leqslant x \wedge\right.\right. & \cdots \wedge v_{s} \leqslant x \\
& \left.\left.\wedge R\left(\phi, v_{1}, \ldots, v_{s}, \bar{b}\right)\right)\right\} .
\end{aligned}
$$

The values of this function on the standard integers $\mathbb{Q}_{0}^{\Re}, \mathbb{Q}_{1}^{\pi}, \ldots$ are standard formulas and constitute an axiomatization of $Q$. We also denote this sequence by $\mathbb{Q}_{0}, \mathbb{Q}_{1}, \ldots, \mathbb{Q}_{m}, \ldots$ The function $y=\mathbb{Q}_{x}$ is easily seen to be $\Delta_{n+1}^{0}$ in the parameter $b$. We also note for future reference that $y=Q_{x}$ is absolute in all $\Pi_{n}^{0}$-elementary extensions of $\Re$ which satisfy $\mathfrak{P}_{n+2}^{0}$.

Let $F(v)$ be a formula of $\mathcal{L}$ and let $a \in|\Re|$. Recall that the formula $F(\bar{a})$ has a "copy" $F(\bar{a})_{\Re}$ in $\Re$. Consider the formula

$$
\forall \phi\left(\operatorname{Tr}_{n}(\phi) \rightarrow \neg \operatorname{Prov}\left(\phi, \mathbb{Q}_{x}, \overline{F(\bar{a})_{\mathscr{R}}} ; \bar{\Lambda}\right)\right) .
$$

The formula (*) has free variable $x$ and parameters $b$ and $F(\bar{a})_{\mathscr{T}}$; moreover, 
this formula expresses that " $Q_{x}+F(\bar{a})$ is consistent with the $\forall^{n}$-theory of the model". We abbreviate (*) by

$$
\operatorname{Cons}\left(T_{n}+Q_{x}+\overline{F(\bar{a})}\right) \text {. }
$$

Let us also remark that, if $\Re \vDash \mathcal{Q}$, then by Theorem 1.6 we have $\Re \vDash$ $\operatorname{Cons}\left(T_{n}+\mathbb{Q}_{\bar{m}}\right)$, for all $m \in \mathbf{N}$. Let us formulate this explicitly:

THEOREM 2.1. Let $\mathbb{Q}$ be an extension of $\mathcal{P}$, let $\mathfrak{T}$ be a model of $\mathbb{Q}$ in which $\mathbb{Q}$ is represented and let $n \geqslant 0$. Then for all standard integers $m$, we have $\mathfrak{N} \vDash$ $\operatorname{Cons}\left(T_{n}+\mathbb{Q}_{\bar{m}}\right)$.

As a corollary to Theorem 2.1 and the Arithmetized Completeness Theorem we have

Theorem 2.2 (Finiteness Lemma). Suppose $\mathfrak{N}$ is a model of $\mathscr{P}$ in which $\mathbb{Q}$ is represented. Let $n \geqslant 0$, let $F(v)$ be a formula and let $a \in|\mathscr{T}|$. Suppose that for all standard integers $m$, we have $\Re \vDash \operatorname{Cons}\left(T_{n}+Q_{\bar{m}}+\overline{F(\bar{a})}\right)$. Then there is a $\Pi_{n}^{0}$-elementary end extension of $\Re$ which satisfies $\mathbb{Q}+F(\bar{a})$.

Proof. If $\mathscr{N}$ is the standard model, this is just the usual Finiteness Lemma or Compactness Theorem. If $\mathscr{T}$ is nonstandard, then $\mathbf{N}$ is not definable in $\mathfrak{N}$; hence there exists a nonstandard integer $k \in|\mathfrak{N}|$ such that $\mathfrak{N} \vDash$ $\operatorname{Cons}\left(T_{n}+\mathbb{Q}_{\bar{k}}+\overline{F(\bar{a})}\right)$. Taking $\phi_{0}$ to be $\left(\mathbb{Q}_{\bar{k}} \wedge F(\bar{a})\right)^{\mathfrak{R}}$ we apply the Arithmetized Completeness Theorem and obtain a $\forall^{n}$-elementary end extension $\Re$ of $\mathscr{R}$ which satisfies $\mathbb{Q}+F(\bar{a})$. Since $\mathcal{Q}$ is an extension of $\mathscr{P}$, the model $\Re$ is thus a $\Pi_{n}^{0}$-elementary extension of $\mathscr{N}$.

In [G], the following question is raised: let $9 \Re$ be a model of $\mathscr{Q}$ and let $P\left(x_{1}, \ldots, x_{s}\right)=Q\left(x_{1}, \ldots, x_{s}\right)$ be a diophantine equation with coefficients from $|\mathfrak{T}|$. Suppose that $P\left(x_{1}, \ldots, x_{s}\right)=Q\left(x_{1}, \ldots, x_{s}\right)$ is solvable in a model of $\mathbb{Q}$ which is an extension of $\mathfrak{T}$; is the equation solvable in a model of $\mathcal{Q}$ which is an end extension of $\Re$ ? The following theorem and its corollaries give a positive solution to this question when the theory $Q$ is represented in $\Re$.

THEOREM 2.3. Suppose $\mathfrak{N}$ is a model of $\mathscr{P}$ in which $\mathbb{Q}$ is represented. Let $n \geqslant 0$, let $a_{1}, \ldots, a_{t} \in|\Re|$ and let $E=E\left(\bar{a}_{1}, \ldots, \bar{a}_{t}\right)$ be a closed formula which is satisfiable in a model of $\mathbb{Q}$ which is $a \forall^{n}$-elementary extension of $\mathfrak{R}$. Then $E$ is satisfiable in a model of $\mathbb{Q}$ which is a $\Pi_{n}^{0}$-elementary end extension of IR.

Proof. Without loss of generality, we may suppose that $E$ is a $\forall^{k}$-sentence with $k \geqslant n$. Recall that $\mathcal{Q}$ is represented in $\mathfrak{N}$ by means of a $\Pi_{0}^{0}$-formula. By the Finiteness Lemma, it is sufficient to show that, for all standard $m$, $\mathfrak{R}$ $\vDash \operatorname{Cons}\left(T_{n}+Q_{\bar{m}}+\bar{E}\right)$. Arguing by contradiction, suppose there exist $d$, $\phi_{0} \in|\Re|$ and standard $m$ such that 


$$
\Re \vDash \operatorname{Tr}_{n}\left[\phi_{0}\right] \text { and } \Re \vDash \operatorname{Prf}\left[d ; \mathbb{Q}_{m},(\neg E)_{\Re}\right] \text {. }
$$

Next let $\Re$ be a $\forall^{n}$-elementary extension of $\Re$ such that $\Re \vDash \mathscr{Q}+E$. By Theorem 1.1, $\Re$ is a $\Pi_{n}^{0}$-elementary extension and we have $\Re \vDash \operatorname{Tr}_{n}\left[\phi_{0}\right]$; since $\operatorname{Prf}\left(u ; v_{1}, \ldots, v_{s}, w\right)$ is $\Pi_{0}^{0}$ it follows from Theorem 1.1 that $\mathscr{A} \vDash$ $\operatorname{Prf}\left[d ; \phi_{0}, \mathbb{Q}_{m} ;(\neg E)_{\Re \pi}\right]$. By Scheme 1.5 , we have $\Re \vDash \operatorname{Tr}_{k}\left[E_{\Im \pi}\right]$. By the predicate calculus this would mean that $\Re \vDash \neg \operatorname{Cons}\left(T_{k}+Q_{\bar{m}}\right)$ which is absurd by virtue of Theorem 2.1 .

In general can the hypothesis that $\mathscr{Q}$ is represented in $\mathscr{N}$ be eliminated in the above theorem? This question is interesting in connection with Theorem 4 of [G].

CoRollary 2.4. Suppose $\mathbb{N}$ is a model of $\mathcal{P}$ in which $\mathbb{Q}$ is represented. Suppose also that there is $a \forall^{n}$-elementary extension of $\mathfrak{T}$ which is a model of $\mathcal{Q}$. Then there is $a \Pi_{n}^{0}$-elementary end extension of $\mathfrak{N}$ which is a model of $\mathbb{Q}$.

Since recursively enumerable sets are represented in all models of $\mathcal{P}$, we have at once

COROLlaRY 2.5. Let $\mathbb{Q}$ be recursively enumerable. If $\mathfrak{T}$ is a model of $\mathcal{P}$ which can be embedded in a model of $\mathcal{Q}$, then $\mathfrak{N}$ has an end extension which is a model of $Q$.

REMARK 2.6. In Corollary 2.4 it is necessary to assume that $\mathbb{Q}$ or the set of theorems of $\mathbb{Q}$ is represented in $\Re$ and in Corollary 2.5 that $Q$ or the set of theorems of $\mathbb{Q}$ is recursively enumerable.

The next corollary is the solution to the problem of Gaifman mentioned above.

CoROLlaRY 2.7. Suppose $\mathfrak{T}$ is a model of $\mathcal{P}$ in which $\mathfrak{Q}$ is represented. Let $a_{1}, \ldots, a_{t} \in|\Re|$ and let $P\left(x_{1}, \ldots, x_{s}, a_{1}, \ldots, a_{t}\right)=$ $Q\left(x_{1}, \ldots, x_{s}, a_{1}, \ldots, a_{t}\right)$ be a diophantine equation which has a solution in a model of $\mathbb{Q}$ which is an extension of $\mathbb{T}$. Then the equation has a solution in a model of $\mathfrak{Q}$ which is an end extension of $\mathfrak{T}$.

In $\S 4$, we use Theorem 2.3 to construct existentially complete models of $\mathbb{G}_{2}^{0}$ which are direct limits of end extension chains of models of $Q$.

REMarks 2.8. In [Ra], Rabin has shown that if $\Re$ is a model of $\mathbb{Q}$, then there is a diophantine equation with coefficients from $\mathscr{N}$ which is not solvable in $\Re$ but which is solvable in an extension of $\Re$ satisfying $\mathcal{Q}$. By Corollary 2.6 this extension can be taken to be an end extension if $\mathbb{Q}$ is represented in $\Re$. Examples of such diophantine equations are given in \$3, cf. Remark 3.10. Furthermore, Wilkie has shown in [W] that for countable T, there always exist such equations that are solvable in an end extension of ก which is isomorphic to $\pi$. 
3. $\forall^{n+1}$-definability of $\mathrm{N}$ in nonstandard models. Let $P_{0}, P_{1}, \ldots$ be a recursive enumeration of the Peano axioms. Set $C_{m}=\bigwedge_{i<m} P_{i}$. As is well known, cf. [K, T], for sufficiently large $m$, the following form of Rosser's Theorem holds: Let $R_{m}$ satisfy

$$
C_{m} \vdash R_{m} \leftrightarrow \forall u\left[\operatorname{Prf}\left(u ; \bar{C}_{m} ; \bar{R}_{m}\right) \rightarrow \exists v\left(v<u \wedge \operatorname{Prf}\left(v ; \bar{C}_{m} ; \neg \bar{R}_{m}\right)\right] .\right.
$$

Then if $C_{m}$ is consistent, $R_{m}$ is independent of $C_{m}$; furthermore, we have $C_{m}$ $\vdash \operatorname{Cons}\left(\bar{C}_{m}\right) \rightarrow R_{m}$ and so $C_{m}+\operatorname{Cons}\left(\overline{C_{m}}\right)$. The following lemma is a more or less straightforward adaptation of these remarks.

LEMMA 3.1. Fix $n \geqslant 0$. Then for sufficiently large $m$, we have

$$
\begin{aligned}
& \mathcal{P} \vdash \forall \psi\left(\operatorname{Cons}\left(T_{n}+\bar{C}_{m}+\psi\right)\right. \rightarrow \\
&\left.\forall \rho\left(\operatorname{Tr}_{n}(\rho) \rightarrow \neg \operatorname{Prov}\left(\bar{C}_{m}, \rho, \psi ; \overline{\operatorname{Cons}}\left(T_{n}+\tilde{\psi}+\tilde{\bar{C}_{m}}\right)\right)\right)\right) .
\end{aligned}
$$

Proof. Let $R(\psi)$ be such that

$$
\begin{aligned}
C_{m} \vdash R(\psi) \leftrightarrow \forall u \forall \rho\left(\operatorname{Tr}_{n}(\rho) \wedge\right. & \operatorname{Prf}\left(u ; \rho, \psi, \bar{C}_{m} ; \bar{R}(\tilde{\psi})\right) \\
& \left.\rightarrow \exists v\left(v<u \wedge \operatorname{Prf}\left(v ; \rho, \psi, \bar{C}_{m} ; \overline{\neg R}(\tilde{\psi})\right)\right)\right) .
\end{aligned}
$$

For $m$ sufficiently large, using Scheme 1.3 and Remark 1.4, we obtain

$$
C_{m} \vdash \forall \psi\left(\operatorname{Cons}\left(T_{n}+\psi+\bar{C}_{m}\right) \rightarrow R(\psi)\right) ;
$$

and carrying out the proof of Rosser's Theorem in $\mathscr{P}$ we have,

$$
\mathcal{P} \vdash \forall \psi\left(\operatorname{Cons}\left(T_{n}+\psi+\bar{C}_{m}\right) \rightarrow \operatorname{Cons}\left(T_{n}+\bar{C}_{m}+\psi+\overline{\neg R}(\tilde{\psi})\right)\right) \text {. }
$$

From (*) and the fact that (*) is itself provable in $C_{m}$ for sufficiently large $m$, together with $(* *)$ we obtain the result.

THEOREM 3.2. Let $n$ be a nonnegative integer, let $\mathfrak{R}$ be a nonstandard model of $\mathbb{Q}$ in which $\mathbb{Q}$ is represented by means of $a \Pi_{n}^{0}$-formula $R\left(x_{1}, y_{1}, \ldots, y_{s}, \bar{b}\right)$ and let $k_{0}$ be an infinite integer of $\Re$. There exists a $\Pi_{n}^{0}$-elementary end extension $\Re$ of $\Re$ such that $\Re \vDash \mathscr{Q}$ and $\Re \vDash \neg \operatorname{Cons}\left(T_{n}+\mathbb{Q}_{\bar{k}_{0}}\right)$.

Proof. We suppose $\Re \vDash \operatorname{Cons}\left(T_{n}+\mathbb{Q}_{\bar{k}_{0}}\right)$. Set $\psi_{0}=\left(\mathbb{Q}_{\bar{k}_{0}}\right)^{\Re}$. Since $\Re \vDash$ $\operatorname{Prov}\left(\mathbb{Q}_{\bar{k}_{0}} ; \bar{C}_{m}\right)$, we can apply the lemma specializing $\psi$ to $\psi_{0}$; hence

$$
\Re \vDash \forall \rho\left(\operatorname{Tr}_{n}(\rho) \rightarrow \neg \operatorname{Prov}\left(\bar{C}_{m}, \bar{\psi}_{0}, \rho ; \overline{\operatorname{Cons}}\left(T_{n}+\widetilde{\bar{\psi}_{0}}+\widetilde{\widetilde{C}_{m}}\right)\right)\right) .
$$

Taking $\phi_{0}$ to be $\left(\bar{\psi}_{0} \wedge \neg \operatorname{Cons}\left(T_{n}+\widetilde{\widetilde{\psi}_{0}}+\tilde{\bar{C}}_{m}\right)\right)^{\Re \pi}$ and applying the Arithmetized Completeness Theorem, there exists a $\Pi_{n}^{0}$-elementary end extension $\mathfrak{\imath}$ 
of $\Re$ such that $\Re \vDash Q \mathcal{Q}+\neg \operatorname{Cons}\left(T_{n}+\bar{\psi}_{0}\right)$. However, since $\Re$ is a $\Pi_{n}^{0}$-elementary extension of $\Re$, the formula $R\left(x, y_{1}, \ldots, y_{s}, \bar{b}\right)$ represents $\mathbb{Q}$ in $\Re$ and the function $y=\mathbb{Q}_{x}$ defined in terms of this representation is absolute. Thus, $\mathscr{T} \vDash \bar{\psi}_{0}=\mathbb{Q}_{\bar{k}_{0}}$ and so $\Re \vdash \mathscr{Q}+\neg \operatorname{Cons}\left(T_{n}+\mathbb{Q}_{\bar{k}_{0}}\right)$.

THEOREM 3.3. Let $\mathbb{T}_{0}$ be a nonstandard model of $\mathfrak{Q}$ in which $\mathfrak{Q}$ is represented by means of $a \Pi_{n}^{0}$-formula $R\left(x, y_{1}, \ldots, y_{s}, \bar{b}\right)$ and let $S$ be an initial segment of $\pi$ such that

(i) $k \in S \Rightarrow \Re \vDash \operatorname{Cons}\left(T_{n}+\mathbb{Q}_{\bar{k}}\right)$,

(ii) $k \in S \Rightarrow$ there exists $l \in S$ such that

$$
\mathfrak{N} \vDash \exists \psi\left(\operatorname{Tr}_{n}(\psi) \wedge \operatorname{Prov}\left(\psi, \mathbb{Q}_{i} ; \quad \overline{\operatorname{Cons}}\left(T_{n}+\tilde{\mathbb{Q}}_{\bar{k}}\right)\right)\right) .
$$

Suppose further that there is a denumerable decreasing sequence $k_{0}>k_{1}$ $>\cdots>k_{s}>\cdots, k_{s} \in|\Re|$, such that

(iii) $k \in S \Leftrightarrow k<k_{s}$, for all $s \in \mathbf{N}$.

Then there exists a $\Pi_{n}^{0}$-elementary end extension chain $\mathfrak{N}_{t}, t \in \mathbb{N}$, of models of $\mathbb{Q}$ with direct limit $\mathfrak{\pi}_{\omega}$ such that $\mathfrak{T}_{\omega} \vDash \mathbb{Q}_{n+2}^{0}$ and $S$ is $\forall^{n+1}$ definable in $\mathfrak{\pi}_{\omega}$ (from parameter $b$ ).

Proof. By (iii), we have $\mathbf{N} \subseteq S$; by Lemma 3.1 and (i) and (ii), $S$ has no greatest element and so $S$ is not definable in $\Re$. Let $T=\left\{k \in\left|\Re_{0}\right|: \Re_{0}=\right.$ Cons $\left.\left(T_{n}+\mathbb{Q}_{\bar{k}}\right)\right\}$. Since $S \subseteq T$ and $T$ is definable in $\mathfrak{T}_{0}$, there must exist $k \in T-S$. As $T$ is also an initial segment of $\Re$, for some $s_{0}$, we have $k_{s_{0}} \in T-S$. By (ii) and (iii), $k \in S$ implies

$$
\mathscr{R}_{0} \vDash \exists \psi\left(\operatorname{Tr}_{n}(\psi) \wedge \operatorname{Prov}\left(\psi, \mathbb{Q}_{\bar{k}_{s_{0}}} ; \overline{\operatorname{Cons}}\left(T_{n}+\tilde{\mathbb{Q}}_{\bar{k}}\right)\right)\right) .
$$

Thus for some $s_{1}>s_{0}$,

$$
\mathscr{R}_{0}=\exists \psi\left(\operatorname{Tr}_{n}(\psi) \wedge \operatorname{Prov}\left(\psi, \mathbb{Q}_{\bar{k}_{s_{0}}} ; \overline{\operatorname{Cons}}\left(T_{n}+\tilde{\mathscr{Q}}_{\bar{k}_{s_{1}}}\right)\right)\right) .
$$

Continuing thus, we can define a cofinal subsequence $l_{t}=k_{s_{1}}$ such that, for all $t \in \mathbf{N}$,

$$
\Re_{0} \vDash \exists \psi\left(\operatorname{Tr}_{n}(\psi) \wedge \operatorname{Prov}\left(\psi, \mathbb{Q}_{\bar{i}} ; \overline{\operatorname{Cons}}\left(T_{n}+\tilde{\mathbb{E}}_{\bar{l}_{+1}}\right)\right)\right) .
$$

By the method of proof of Theorem 3.2, there exists a $\Pi_{n}^{0}$-elementary end extension $\Re_{1}$ of $\mathbb{M}_{0}$ such that $\mathbb{R}_{1} \vDash \mathbb{Q}+\tau_{7} \operatorname{Cons}\left(T_{n}+\mathbb{Q}_{i_{0}}\right)+\operatorname{Cons}\left(T_{n}+\right.$ $\left.\mathbb{Q}_{i_{1}}\right)$. Iterating this step, we can construct a $\Pi_{n}^{0}$-elementary end extension chain $\Re_{t}, t \in N$, such that

$$
\left.\mathscr{N}_{t} \vDash \mathscr{Q}+\right\urcorner_{7} \operatorname{Cons}\left(T_{n}+\mathbb{Q}_{\bar{l}}\right)+\operatorname{Cons}\left(T_{n}+\mathbb{Q}_{\bar{l}_{i+1}}\right) .
$$

Let $\Re_{\omega}$ be the direct limit of this chain. Then $\mathfrak{N}_{\omega}$ is a $\Pi_{n}^{0}$-elementary end extension of each $\mathfrak{R}_{t}$ and each $\mathfrak{R}_{t} \vDash \mathbb{Q}$; therefore $\mathfrak{K}_{\omega} \vDash \mathbb{Q}_{n+2}^{0}$. Moreover, 
if $k \in\left|\Re_{\omega}\right|$ and $k \notin S$, then for some $t, l_{t}<k$ and so $\Re_{t+1} \vDash \neg \operatorname{Cons}\left(T_{n}\right.$ $\left.+\mathbb{Q}_{\bar{k}}\right)$; since $\neg \operatorname{Cons}\left(T_{n}+\mathbb{Q}_{\bar{k}}\right)$ is a $\Sigma_{n+1}^{0}$-sentence (with parameters $\bar{b}$ and $\bar{k}$ ), the limit model $\mathfrak{N}_{\omega}$ satisfies $\neg \operatorname{Cons}\left(T_{n}+\mathbb{Q}_{\bar{k}}\right)$. On the other hand, for $k \in S$, we have $\mathfrak{T}_{t} \vDash \operatorname{Cons}\left(T_{n}+\mathbb{Q}_{\bar{k}}\right)$ for all $t$ and so $\mathfrak{T}_{\omega} \vDash \operatorname{Cons}\left(T_{n}+\right.$ $\left.\mathbb{Q}_{\bar{k}}\right)$. We therefore obtain for $k \in\left|\bigcap_{\omega}\right|$,

$$
k \in S \Leftrightarrow \Re_{\omega} \vDash \forall x\left(x<\bar{k} \rightarrow \operatorname{Cons}\left(T_{n}+\mathbb{Q}_{x}\right)\right) .
$$

The formula $\forall x\left(x<y \rightarrow \operatorname{Cons}\left(T_{n}+\mathbb{Q}_{x}\right)\right)$ is $\Pi_{n+1}^{0}$ with parameter $\bar{b}$ and free variable $y$; let us abbreviate this formula further by $H(y, \bar{b})$. By Matiasevitch's work, there is a $\forall^{n+1}$-formula $F(y, z)$ such that $\mathscr{P} \vdash \forall y$ $\forall z(H(y, z) \leftrightarrow F(y, z))$. Since this equivalence is $\Pi_{n+2}^{0}$ and provable in $\mathcal{P}$, it holds in $\mathfrak{T}_{\omega}$. So the initial segment $S$ is definable in $\mathfrak{N}_{\omega}$ as $\left\{k: \Re_{\omega} \vDash\right.$ $F(\bar{k}, \bar{b})\}$.

Let us call a model $\Re$ initially countable if $|\Re|$ is countable or if for some nonstandard $k \in|\Re|$, the set $\{l: \Re \models \bar{l}<\bar{k}\}$ is countable.

CoRollary 3.4. Let $\mathfrak{T}_{0}$ be an initially countable model of $\mathbb{Q}$ in which $\mathfrak{Q}$ is represented by means of $a \Pi_{n}^{0}$-formula $R\left(u, v_{1}, \ldots, v_{s}, \bar{b}\right)$. Then there exists a proper $\Pi_{n}^{0}$-elementary end extension chain $\Re_{t}, t \in \mathbf{N}$, of models of $\mathbb{Q}$ such that in the limit model $\Re_{\omega}=\cup_{t \in \mathrm{N}} \Re_{t}$ the standard integers are definable by $a$ $\forall^{n+1}$-formula (with parameter $b$ ).

Proof. We can assume $\mathfrak{T}_{0}$ is nonstandard. We then apply Theorem 3.3 with $S=\mathrm{N}$ : conditions (i) and (ii) are met by virtue of Theorem 2.1; condition (iii) is met since $\Re_{0}$ is initially countable.

Remark 3.5. The case where $\Re_{0}$ is not initially countable is treated in $\S \S 5$ and 6 of this paper.

In [Ry], Ryll-Nardzewski established that $\mathscr{P}$ is not finitely axiomatizable. The next two corollaries yield independence results of this kind which are optimal from the point of view of quantifier complexity; they thus strengthen results that have been obtained either proof-theoretically or model theoretically by various authors; cf., for example, $[\mathbf{K}, \mathbf{L}],[\mathbf{G}, \mathbf{M}, \mathbf{S}]$ and $[\mathbf{W}]$ which are recent contributions to the literature. Some further results of this kind are given in $\$ 6$ of this paper.

Corollary 3.6. Let $\mathbb{Q}$ be a consistent extension of $\mathcal{P}$. Then there exists $\forall^{n+3}$-sentence which is the prenex form of an instance of the induction scheme and which is independent of $\mathbb{E}_{n+2}^{0}$.

Proof. Let $\mathbb{N}_{0}$ be a countable nonstandard model of $\mathscr{Q}$ in which $\mathbb{Q}$ is represented by a $\Pi_{0}^{0}$-formula $R(x, \bar{b})$. Let $\Re_{\omega}$ and $F(y, \bar{b})$ be as in the proof of Corollary 3.3 above so that the standard integers are definable in $\mathfrak{N}_{\omega}$ by the $\forall^{n+1}$-formula $F(y, \bar{b})$. Then $\Re_{\omega} \models \mathbb{Q}_{n+2}^{0}$ but the following instance of the induction scheme fails in $\mathfrak{R}_{\omega}$ : 


$$
\forall z((F(0, z) \wedge \forall y(F(y, z) \rightarrow F(y+1, z))) \rightarrow \forall y F(y, z)) .
$$

Since $F(y, z)$ is $\forall^{n+1}$, the prenex form of this instance of the induction scheme is $\forall^{n+3}$.

Corollary 3.7. Suppose that $\mathfrak{N}_{0}$ is a countable model of $\mathbb{Q}$ in which $\mathbb{Q}$ is represented by a $\Pi_{n}^{0}$-formula $R\left(x, y_{1}, \ldots, y_{s}\right)$ without parameters. Let $\mathfrak{B}$ be the set of $\Sigma_{n+1}^{0}$ formulas satisfied in $\mathfrak{T}_{0}$. Then there exists a $\exists^{n+2}$ sentence which is the prenex form of an instance of the induction scheme and which is independent of $(\mathbb{Q} \cup \mathscr{B})_{n+2}^{0}$.

Proof. We follow the notation of the proof of Theorem 3.3: let $\Re_{\omega}$ be the limit model and let $F(y)$ be the parameter free $\forall^{n+1}$-formula which defines $\mathbf{N}$ in $\mathfrak{T}_{\omega}$. Then $\mathfrak{K}_{\omega} \vDash(Q \cup \mathscr{B})_{n+2}^{0}$ but the following instance of the induction scheme fails in $\mathfrak{T}_{\omega}:(F(0) \wedge \forall y(F(y) \rightarrow F(y+1))) \rightarrow \forall y F(y)$.

The following corollaries generalize a result of Hirschfeld, cf. $[\mathbf{H}, \mathbf{W}$, Chapter 8]; also cf. $\$ \S 5$ and 6 of this paper.

CoROllaRY 3.8. Let $\mathscr{B}$ be a set of $\Pi_{2}^{0}$-sentences which is consistent with $\mathscr{P}$. There exist a diophantine equation $P\left(x, z, y_{1}, \ldots, y_{m}\right)=Q\left(x, z, y_{1}, \ldots, y_{m}\right)$, a nonstandard model $\Re$ of $\mathscr{B}$ and an element $b \in|\Re|$ such that, for $k \in|\Re|$,

$$
k \notin \mathbf{N} \Leftrightarrow \Re \vDash \exists y_{1} \ldots \exists y_{m}\left(P\left(\bar{k}, \bar{b}, y_{1}, \ldots, y_{m}\right)=Q\left(\bar{k}, \bar{b}, y_{1}, \ldots, y_{m}\right)\right) \text {. }
$$

For recursively enumerable $\mathscr{B}$ the parameter $b$ disappears and we have

COROLlaRY 3.9. Let $\mathscr{B}$ be a recursively enumerable set of $\Pi_{2}^{0}$-sentences which is consistent with $\mathcal{P}$. There exist a diophantine equation $P\left(x, y_{1}, \ldots, y_{m}\right)$ $=Q\left(x, y_{1}, \ldots, y_{m}\right)$ and $a$ nonstandard model $\Re$ of $\mathscr{B}$ such that, for all $k \in|\Re|$,

$$
k \notin \mathbf{N} \Leftrightarrow \Re \vDash \exists y_{1} \ldots \exists y_{m}\left(P\left(\bar{k}, y_{1}, \ldots, y_{m}\right)=Q\left(\bar{k}, y_{1}, \ldots, y_{m}\right)\right) .
$$

REMARK 3.10. Suppose that $\mathscr{T}$ is a model of $\mathbb{Q}$ and that $\mathbb{Q}$ is represented in $\Re$. If $\Re \vDash \operatorname{Cons}\left(\mathbb{Q}_{\bar{k}}\right)$ with $k$ infinite, then by Matijasevich's work and Theorem 3.2, $\neg \operatorname{Cons}\left(\mathbb{Q}_{\bar{k}}\right)$ yields an example of a diophantine equation which is not solvable in $\mathfrak{T}$ but which is solvable in an end extension. (Such equations were first constructed by Rabin, cf. Remarks 2.8.) This example is also noted by Manewitz in [Man] for the case $\mathbb{Q}$ recursively enumerable and $\Re \vDash Q+\forall x \operatorname{Cons}\left(\mathbb{Q}_{x}\right)$.

4. Recursively enumerable theories. In this section we concentrate on recursively enumerable theories $\mathscr{Q}$ and examine some nuances of the Second Incompleteness Theorem. Associated with a $\Pi_{0}^{0}$-representation $R\left(u, v_{1}, \ldots, v_{s}\right)$ of $\mathbb{Q}$ in $\mathbf{N}$ we have a consistency statement $\forall x \operatorname{Cons}\left(\mathbb{Q}_{x}\right)$; this $\Pi_{1}^{0}$-sentence depends on $R$ and we denote it by $\operatorname{Cons}_{R}(Q)$. It is shown in 
[Fef] that the strength of this formula varies considerably with the choice of $R$. It follows from Theorem 3.2 that for fixed $R$, if $\Re$ is a model of $\mathcal{Q}$, then $\mathfrak{N}$ has an end extension satisfying $\mathscr{Q}+\neg \operatorname{Cons}_{R}(\mathfrak{Q})$; this is in fact Theorem 6.6 of [Fef]. One might ask whether $\Re$ has an extension or end extension which satisfies $\mathbb{Q}+\neg \operatorname{Cons}_{R}(\mathbb{Q})$ for all $R$. On the one hand, there is a "positive" result, viz:

THEOREM 4.1. Let $\mathscr{Q}$ be a recursively enumerable extension of $\mathcal{P}$ and let $\mathfrak{T}$ be a model of $\mathcal{Q}$. Then there exists an extension $\Re$ of $\mathfrak{N}$ such that $\mathfrak{N} \neq \mathbb{Q}$ and $\Re \vDash \neg \operatorname{Cons}_{R}(\mathbb{Q})$ for all $\Pi_{0}^{0}$-parameter free representations $R\left(u, v_{1}, \ldots, v_{s}\right)$ of $\mathbb{Q}$.

Proof. Let $R_{0}, R_{1}, \ldots$ be an enumeration of the $\Pi_{0}^{0}$-representations of $\mathscr{Q}$ in N. By the Compactness Theorem, there is an elementary extension $\mathfrak{R}^{*}$ of T with nonstandard elements $b$ and $l_{s t}, s, t \in \mathrm{N}$, such that

$$
\mathfrak{T}^{*} \vDash \operatorname{Prov}\left(\mathbb{Q}_{T_{u}^{\prime}}^{R}, \mathbb{Q}_{\bar{b}}^{R}\right)
$$

where $y=\mathbb{Q}_{x}^{R}$ is the function $y=\mathbb{Q}_{x}$ is determined by $R$. Next we apply Lemma 3.1 to obtain an end extension $\Re$ of $\pi *$ such that $\Re \vDash \mathbb{Q}+$ $\neg$ Cons $\left(\mathbb{Q}_{b}^{R_{0}}\right)$. We then necessarily have $\mathscr{\neg} \neg \operatorname{Cons}_{R_{9}}(\mathbb{Q})$ for all $s \in N$.

In the language of [Fef], we have shown

THEOREM 4.1a. Let $\mathbb{Q}$ be a recursively enumerable extension of $\mathcal{P}$ and let $\mathfrak{R}$ be a model of $\mathbb{Q}$. There is an extension $\Re$ of $\Re$ such that $\Re \vDash \mathbb{Q}$ and for all RE-numerations $\alpha$ of $\mathcal{Q}, \vartheta \mathcal{\tau}=\neg$ Cons $_{\alpha}$.

On the other hand, there is a "negative" result; however this will require some definitions and an auxiliary theorem.

Definition 4.2. Let $\Re$ be a nonstandard model of $\mathcal{P}$. We say that a sequence $\left(k_{i}\right), i \in \mathrm{N}$, of nonstandard integers is downward cofinal iff for all $l \in|\Re|, l$ is nonstandard $\Leftrightarrow$ for some $i, \Re \models \bar{l}>\bar{k}_{i}$.

Definition 4.3. Let $\Re$ be a model of $\mathscr{P}$ and let $k \in|\mathfrak{T}|$. We say that $k$ is a $\Pi_{n}^{0}$-minimal element if for some parameter free $\Pi_{n}^{0}$-formula $E(x)$, $\Re \vDash$ $E(\bar{k}) \wedge \forall x(x<\bar{k} \rightarrow \neg E(x))$.

THEOREM 4.4. Suppose that $\mathbb{Q}$ is consistent with $\mathfrak{F}_{n}^{0}$ and that $\mathbb{Q}$ is represented in $\mathbf{N}$ by a $\Pi_{n}^{0}$-formula $R\left(u, v_{1}, \ldots, v_{i}\right)$. Then $\mathbb{Q}$ has a nonstandard model $\Re$ with a downward cofinal sequence of $\Pi_{n}^{0}$-minimal elements.

Proof. The model $\Re$ is constructed using the Henkin method of proof of the Completeness Theorem. Note that we can assume without loss of generality that $\mathbb{Q} \vdash \mathscr{T}_{n}^{0}$ and also that $\mathscr{Q} \vdash \exists x \neg \operatorname{Cons}_{R}\left(T_{n}+\mathbb{Q}_{x}\right)$. These assumptions guarantee that all models of $\mathscr{Q}$ are nonstandard $\Pi_{n}^{0}$-elementary extensions of $\mathbf{N}$. The theorem is then a straightforward application of the following lemma. 
LeMma 4.5. Let $c_{1}, \ldots, c_{s+1}$ be new individual constants and let $t \leqslant s$. Suppose that $\mathbb{Q}+F\left(c_{1}, \ldots, c_{s}\right)$ is consistent and that $\mathbb{Q}+F\left(c_{1}, \ldots, c_{s}\right) \vdash$ $c_{t}>\bar{m}$ for all standard $m$. Then there is a parameter free $\Pi_{n}^{0}$-formula $E(u)$ such that $\mathbb{Q}+F\left(c_{1}, \ldots, c_{s}\right)+E\left(c_{s+1}\right)+c_{s+1} \leqslant c_{t}$ is consistent and $\mathbb{Q}-$ $\neg E(\bar{m})$ for all standard $m$.

Proof of the lemma. Let $\exists z G(x, y, z)$ be a parameter free $\Sigma_{n+1}^{0}$ formula which defines the function $y=\mathbb{Q}_{x}^{R}$ in $\Re$. Let $S$ be a closed formula which satisfies

$$
\begin{aligned}
\mathcal{Q} \vdash S \leftrightarrow \forall x \forall y \forall z \forall w[G(x, y, z) & \wedge \operatorname{Prf}\left(w ; y, \overline{\exists y_{1} \cdots \exists y_{s} F} ; \bar{S}\right) \\
\rightarrow & \exists y_{1} \cdots \exists y_{s}\left(F\left(y_{1}, \ldots, y_{s}\right)\right. \\
& \left.\left.\wedge\left(y_{t}<w \vee y_{t}<z \vee y_{t}<x \vee y_{t}<y\right)\right)\right] .
\end{aligned}
$$

We claim that $\mathbb{Q}+F\left(c_{1}, \ldots, c_{s}\right)+\neg S$ is consistent: for otherwise, there are standard integers $p, j, m$ such that $\mathbf{N} \models G\left[m, \mathbb{Q}_{m}, j\right]$ and $p$ is a proof of $S$ from the hypotheses $\mathbb{Q}_{m}$ and $\exists y_{1} \cdots \exists y_{s} F$. Let $\Re$ be a model of $\mathbb{Q}+$ $\exists y_{1} \cdots \exists y_{s} F$. Since $\mathbb{Q} \vdash \mathscr{J}_{n}^{0}$, we have $\Re \vDash G\left[m, \mathbb{Q}_{m}, j\right]$ and $\Re \vDash$ $\operatorname{Prf}\left[p ; \mathbb{Q}_{m}, \exists y_{1} \cdots \exists y_{s} F ; S\right]$; hence since $\Re \vDash S$, we obtain

$\Re \vDash \exists y_{1} \cdots \exists y_{s}\left[F\left(y_{1}, \ldots, y_{s}\right) \wedge\left(y_{t}<\bar{p} \vee y_{t}<\bar{m} \vee y_{t}<\overline{\mathbb{Q}}_{m} \vee y_{t}<\bar{j}\right)\right]$

However, this contradicts the hypothesis that $Q+F\left(c_{1}, \ldots, c_{s}\right) \vdash \bar{m}<c_{t}$ for all standard $m$; so the claim is established. We can now take $E(u)$ to be a $\Pi_{n}^{0}$-formula equivalent in $\mathcal{P}$ to

$$
\begin{aligned}
\exists x<u \exists y< & <\exists z<u \exists w \\
& <u\left[G(x, y, z) \wedge \operatorname{Prf}\left(w ; y, \overline{\exists y_{1} \cdots \exists y_{s} F} ; \bar{S}\right)\right] .
\end{aligned}
$$

The "negative" result now follows easily from Theorem 4.4.

THEOREM 4.6. Let $\mathbb{Q}$ be recursively enumerable and let $\mathbb{T}$ be a nonstandard model of $\mathbb{Q}$ with a downward cofinal sequence of $\Pi_{0}^{0}$-minimal nonstandard integers. Then for every end extension $\Re$ of $\Re$ such that $\Re \vDash \mathbb{Q}$ we have $\Re$ $\vDash \operatorname{Cons}_{R}(\mathbb{Q})$ for some $\Pi_{0}^{0}$-parameter free representation of $\mathcal{Q}$.

Proof. Let $S\left(u, v_{1}, \ldots, v_{s}\right)$ be a $\Pi_{0}^{0}$-parameter free representation of $\mathcal{Q}$. Since $\Re \vDash \mathbb{Q}$, for some $\Pi_{0}^{0}$-minimal nonstandard integer $l \in|\Re|$ we have $\Re \vDash \operatorname{Cons}\left(\mathbb{P}_{l}^{S}\right)$. Let $E(x)$ be $\Pi_{0}^{0}$ and such that $\Re \vDash E(\bar{l}) \wedge \forall x(x<\bar{l} \rightarrow$ $\neg E(x))$ and set

$$
\begin{aligned}
R\left(u, v_{1}, \ldots, v_{s}\right) & \equiv S\left(u, v_{1}, \ldots, v_{s}\right) \wedge \forall x<u \forall u_{1}<v_{1} \cdots \forall u_{s} \\
& <v_{s}\left(\neg E(x) \wedge \neg E\left(u_{1}\right) \wedge \cdots \wedge \neg E\left(u_{s}\right)\right) .
\end{aligned}
$$


Formulating the above in the terminology of [Fef], we have

THEOREM 4.6a. Let $\mathbb{Q}$ be recursively enumerable and let $\Re$ be a nonstandard model of $\mathbb{Q}$ with a downward cofinal sequence of $\Pi_{0}^{0}$-minimal nonstandard integers. Then for every end extension $\Re$ of $\Re$ such that $\Re \vDash \mathbb{Q}$, we have $\Re$ $\vDash$ Cons $_{\alpha}$ for some $\mathrm{RE}$-numeration $\alpha$ of $\mathbb{Q}$.

Definition 4.7. Let $\Re$ be a model of $\mathscr{P}$ and let $k \in|\mathfrak{T}|$. We say that $k$ is existentially (resp. universally) definable in $\Re$ iff $\Re \vDash F(\bar{k}) \wedge \exists ! x F(x)$ for some $\exists^{1}$ (resp. $\forall^{1}$ ) parameter free formula $F(x)$.

Since $\Pi_{0}^{0}$-formulas define (provably) recursive sets, cf. [G], we have as a corollary to Theorem 4.4

THEOREM 4.8. Let $\mathscr{Q}$ be a recursively enumerable extension of $\mathcal{P}$. Then $\mathbb{Q}$ has a nonstandard model ๆ with a downward cofinal sequence of infinite elements which are both universally and existentially definable in $\Re$.

5. Existentially complete structures. The model-theoretic notion of existentially complete structure is a generalization of the classical notion of algebraically closed structure, cf. $[\mathbf{H}, \mathbf{W}]$ or $[\operatorname{Sim}]:$ let $\mathcal{C}$ be a first order theory and let $C_{\forall}$ denote the set of universal sentences provable in $C_{\text {; a model }} \Re$ of $C_{\forall}$ is said to be existentially complete iff every existential formula with parameters in $|\mathfrak{T}|$ which is satisfiable in an extension of $\mathscr{N}$ which is a model of $\mathcal{C}$ is already satisfied in $\Re$. It is easy to see that an existentially complete model of $\mathcal{C}_{\forall}$ is in fact a model of $\mathcal{C}_{\forall \exists}$. By standard model-theoretic techniques, one can show that every model of $C$ can be embedded in an existentially complete model of $\mathcal{C}_{\forall \exists}$ (of the same cardinality modulo $\kappa_{0}$ ). The following result answers a question of $\mathrm{G}$. Sabbagh:

THEOREM 5.1. Let $\mathfrak{R}$ be a countable model of $\mathcal{P}$ in which $\mathfrak{Q}$ is represented. Suppose that $\Re$ can be embedded in a model of $\mathcal{Q}$. Then $\mathfrak{N}$ has an end extension which is an existentially complete model of $\mathbb{Q}_{2}^{0}$.

Proof. We can suppose that $\Re$ is not the standard model; we can also suppose by Corollary 2.5 that $\mathscr{N}$ is a model of $\mathcal{Q}$ and by the remarks on page 257 that $\mathscr{Q}$ is represented in $\mathfrak{N}$ by means of a $\Pi_{0}^{0}$-formula. By iterated application of Corollary 2.7, we can construct an end extension chain $\Re_{s}$, $s \in \mathbf{N}$, with $\mathfrak{R}=\mathbb{N}_{0}$, of countable models of $\mathbb{Q}$ with the following property:

For every $s$, for every $a_{1}, \ldots, a_{t} \in\left|\Re_{s}\right|$ and for every existential formula $E\left(\bar{a}_{1}, \ldots, \bar{a}_{t}\right)$, there is an $s^{\prime} \geqslant s$ such that if $E\left(\bar{a}_{1}, \ldots, \bar{a}_{t}\right)$ is satisfiable in some extension of $\Re_{s}$, then $E\left(\bar{a}_{1}, \ldots, \bar{a}_{t}\right)$ is satisfied in $\Re_{s^{\prime}+1}$.

Since this chain is an end extension chain the direct limit is a model of $\mathbb{Q}_{2}^{0}$ and by construction $\Re_{\omega}$ is existentially complete.

The set of sentences satisfied in all existentially complete models of $\mathcal{C}_{\forall}$ is 
denoted $\mathcal{E}(\mathcal{C})$. For consistent extensions $\mathbb{Q}$ of Peano arithmetic, Hirschfeld has shown, cf. [H, W, Chapter 8], that $\mathbb{C}_{2}^{0} \subseteq \mathcal{E}(\mathfrak{Q})$. In the course of the proof it is established that existentially complete models of $\mathscr{Q}$ are in fact " $\Sigma_{1}^{0}$-complete":

Lemma 5.2. Let $\Re$ be an existentially complete model of $Q_{r}$ and let $F\left(v_{1}, \ldots, v_{s}, \bar{a}_{1}, \ldots, \bar{a}_{t}\right)$ be $a \Pi_{0}^{0}$-formula with $a_{1}, \ldots, a_{t} \in|\Re|$. Then, if $\exists v_{1} \cdots \exists v_{s} F\left(v_{1}, \ldots, v_{s}, \bar{a}_{1}, \ldots, \bar{a}_{t}\right)$ is satisfiable in an extension of $\Re$ which is a model of $\mathcal{Q}$, it is satisfiable in $\Re$.

Hirschfeld has also shown

THEOREM 5.3. Let $\mathfrak{Q}$ be a complete extension of $\mathcal{P}$. Then $N$ is $\forall^{1}$-definable (with parameters) in all existentially complete models of $\mathbb{Q}_{2}^{0}$.

Lemma 5.2 and the results of $\$ 3$ yield a complementary result, which, in particular, covers the case of recursively enumerable theories.

THEOREM 5.4. Let $\mathfrak{T}$ be an existentially complete model of $\mathbb{Q}_{2}^{0}$ in which $\mathfrak{Q}$ is represented by means of $a \Pi_{0}^{0}$-formula $R\left(u, v_{1}, \ldots, v_{s}, \bar{b}\right)$. Then the standard integers are definable in $\mathfrak{T}$ by means of $\forall^{1}$-formula (with parameter $b$ ).

Proof. Consider the $\Sigma_{1}^{0}$-formula (with parameter $b$ )

$$
I(x) \equiv \exists y\left(y<x \wedge \neg \operatorname{Cons}\left(\mathbb{Q}_{y}\right)\right) \text {. }
$$

By Theorems 1.6 and 3.2, we have for all $k \in|\Re|, \Re \vDash I(\bar{k}) \Leftrightarrow k$ is nonstandard. Since $\pi \vDash \mathbb{P}_{2}^{0}$, the formula $I(x)$ is equivalent in $\pi$ to an existential formula (with parameter $b$ ).

Since the standard integers can be $\Pi_{1}^{0}$-definable in nonstandard models of $\mathbb{Q}_{2}^{0}, \Sigma_{1}^{0}$-induction (in its least element form) is thus not a consequence of $\mathbb{Q}_{2}^{0}$ nor of $\mathcal{E}(\mathbb{Q})$; and for recursively enumerable $\mathscr{Q}$, even induction for parameter free $\Sigma_{1}^{0}$-formulas fails in all existentially complete models of $\mathcal{Q}$. Something can also be said about $\Pi_{1}^{0}$-induction. If $\Re$ is a model of $\mathscr{P}_{2}^{0}$ and if $a \in|\Re|$, let us denote by $S_{a}^{\Re}$ the set $\{b \in|\Re|:$ for all standard $n, \Re \vDash \bar{b}+\bar{n}<$ $\bar{a}$ \}. Suppose that $\mathfrak{T}$ is an existentially complete model of $\mathbb{Q}_{2}^{0}$ in which $\mathbb{Q}$ is represented by means of $\Pi_{0}^{0}$-formula. For infinite $a \in|\Re|$, consider the formula

$$
J_{a}(x) \equiv x<\bar{a} \wedge \exists y\left(y<\bar{a}-x \wedge \neg \operatorname{Cons}\left(\mathbb{Q}_{y}\right)\right) .
$$

Clearly $J_{a}(x)$ defines $S_{a}^{\Re}$ in $\Re$ and hence $S_{a}^{\Re}$ is $\Sigma_{1}^{0}$-definable (with parameter) in $\Re$. We can conclude that induction for $\Pi_{1}^{0}$ formulas (in its minimal element form) is not a consequence of $\mathscr{E}(\mathbb{Q})$. (This answers a question of Hirschfeld.) For general $\mathbb{Q}$ and $a$, the formula $J_{a}(x)$ will contain parameters from $|\Re|$. However, for recursively enumerable $\mathbb{Q}$ and for certain $a \in|\Re|$, the parameters can be dispensed with: let $a_{0}$ be an infinite $\Pi_{0}^{0}$-minimal 
element of $|\Re|$; such $a_{0}$ exists since $\Re \vDash \exists x \neg \operatorname{Cons}\left(Q_{x}\right)$. Then the formula $J_{a}(x)$ is equivalent in $\Re$ to an existential formula without parameters.

The following definition generalizes the notion of existentially complete models, cf. [Sim].

Definition 5.5. Let $n \geqslant 0$ and let $\Re$ be a model of $\mathcal{C}_{\forall n}$ which is a $\forall \forall^{n}$-elementary subsystem of a model of $e$. Then $\Re \in E_{n}(e)$ iff for all models $\Re$ of $\mathcal{C}, \pi<{ }_{n} \Re \Rightarrow \Re<_{n+1} \Re$.

Results analogous to those above go through for structures in $E_{n}(\mathbb{Q})$ mutatis mutandis. We now state a composite result which will be useful in $\$ 7$.

THEOREM 5.6. Let $\mathfrak{T}_{0}$ be a countable nonstandard model of $\mathbb{Q}$ in which $\mathfrak{Q}$ is represented by means of $a \Pi_{n}^{0}$-formula $R\left(u, v_{1}, \ldots, v_{s}, \bar{b}\right)$ where $n>0$. Then there is a $\Pi_{n}^{0}$-elementary end extension chain starting at $\mathfrak{\pi}_{0}$ with limit model $\mathfrak{T}_{\omega}$ such that (i) $\mathfrak{\pi}_{\omega} \models \mathbb{Q}_{n+2}^{0}$, (ii) $\Re_{\omega} \in E_{n}(\mathbb{Q})$ (iii) the standard integers are $\Pi_{n+1^{-}}^{0}$ definable in $\mathfrak{T}_{\omega}$ as $\left\{x: \operatorname{Cons}\left(T_{n}+\mathbb{Q}_{x}\right)\right\}$.

6. $\Delta_{n+1}^{0}$ definability of $\mathbf{N}$ in nonstandard models. Let us take another look at Theorem 3.3 and consider the case where the sequence $k_{0}>k_{1}>\cdots$ of condition (iii) is itself an initial segment of a sequence in $\mathfrak{N}$; more precisely, let us suppose that there is a sequence number $l$ in $\Re$ whose domain is an infinite integer such that $k_{s}=(l)_{s}$ for all $s \in \mathbf{N}$. Under this additional assumption, in the limit model $\mathfrak{N}_{\omega}$ the standard integers will be definable as $\left\{x: \mathfrak{M}_{\omega}=\neg \operatorname{Cons}\left(T_{n}+\mathscr{Q}_{(l)_{x}}\right)\right\}$; and so the standard integers will be $\exists^{n+1}$. definable in $\Re_{\omega}$. This remark will be used in the proof of Theorem 6.1 which is the main result of this section. For this theorem we will also require a construction of mutually independent formulas that goes back to work of Mostowski; cf. [Mos, bis]; we now outline the elegant presentation of such a construction given by $\mathrm{H}$. Friedman in [ $\mathrm{Fr}]$.

Let $\mathbb{Q}_{0}, \mathbb{Q}_{1}, \ldots$ be an axiomatization of $\mathbb{Q}$; set $C_{m}=\aleph_{i<m} \mathbb{Q}_{i}$ and let $m$ be sufficiently large. Following Friedman, we define a sequence of $\Pi_{n+1^{-}}^{0}$ formulas $\mu_{0}^{m}, \mu_{1}^{m}, \ldots$ with the property that

$$
\begin{aligned}
& C_{m} \vdash \mu_{i}^{m} \leftrightarrow \forall x \forall \psi \forall \rho\left[\left(\operatorname{Tr}_{n}(\psi) \wedge \text { “ } \rho\right.\right. \text { is a disjunction of the } \\
& \text { form } \left. \pm \mu_{0}^{m} \vee \cdots \vee \pm \mu_{i-1}^{m} \wedge \wedge \operatorname{Prf}\left(x ; \bar{C}_{m}, \psi ; \rho \vee \bar{\mu}_{i}\right)\right) \rightarrow \exists y \\
& \left.<x \exists \phi<x\left(\operatorname{Tr}_{n}(\phi) \wedge \operatorname{Prf}\left(y ; \bar{C}_{m}, \phi ; \rho \vee \bar{\neg} \mu_{i}\right)\right)\right]
\end{aligned}
$$

In Lemma 3.3.1 of [Fr], it is shown that all combinations $\pm \mu_{0}^{m} \wedge \cdots \wedge$ $\pm \mu_{j}^{m}$ are consistent with $\mathscr{T}_{n}^{0}+C_{m}$, provided $\mathscr{T}_{n}^{0}+C_{m}$ is consistent.

Let us introduce truth definitions $\operatorname{Tr}_{k}^{*}(v)$ for $\Pi_{k}^{0}$-sentences; for $k=0$, $\operatorname{Tr}_{k}^{*}(v)$ is a $\Delta_{1}^{0}$-predicate and for $k>0, \operatorname{Tr}_{k}^{*}(v)$ is $\Pi_{k}^{0}$. With $m$ sufficiently large, we then have the scheme

$$
C_{m} \vdash F \leftrightarrow \operatorname{Tr}_{n}^{*}(\bar{F}), \quad F \text { a } \Pi_{n}^{0} \text {-sentence. }
$$

Since the function $\left(C_{m, i}\right) \rightarrow \mu_{i}^{m}$ is recursive, we can define 


$$
\begin{aligned}
M_{n}(x, y) & \equiv \operatorname{Tr}_{n+1}^{*}\left(\tilde{\mu} y_{y}^{x}\right), \\
S_{n}(x, y) & \equiv \forall i<y M_{n}(x, i) \wedge \neg M_{n}(x, y) .
\end{aligned}
$$

Friedman's lemma then yields: if $\mathfrak{T}_{n}^{0}+C_{m}$ is consistent, so is $\mathfrak{T}_{n}^{0}+C_{m}+$ $S_{n}(\bar{m}, \bar{k})$ for all integers $k$; the proof also shows that $\mathscr{T}_{n}^{0}+C_{m}+$ $\exists y S_{n}(\bar{m}, y) \vdash \neg \operatorname{Cons}\left(T_{n}+\overline{C_{m}}\right)$.

In our notation, if $\mathscr{N}$ is a model of $\mathscr{Q}$ in which $\mathscr{Q}$ is represented, then for all sufficiently large standard integers $m_{0}$,

$$
\begin{aligned}
\mathscr{N} \vDash \forall x\left(x \geqslant \bar{m}_{0} \rightarrow(\right. & \operatorname{Cons}\left(T_{n}+\mathfrak{Q}_{x}\right) \\
& \left.\left.\rightarrow \forall y \forall \psi\left(\operatorname{Tr}_{n}(\psi) \rightarrow \neg \operatorname{Prov}\left(\psi, \mathcal{Q}_{x}, \bar{S}_{n}(\tilde{x}, \tilde{y}) ; \bar{\Lambda}\right)\right)\right)\right)
\end{aligned}
$$

and

$$
\mathscr{N} \vDash \forall x\left(x \geqslant \bar{m}_{0} \rightarrow\left(\operatorname{Cons}\left(T_{n}+\mathbb{Q}_{x}\right) \rightarrow \forall y M_{n}(x, y)\right)\right) .
$$

THEOREM 6.1. Let $\Re$ be a countable model of $\mathscr{Q}$ in which $\mathfrak{Q}$ is represented by means of $a \Pi_{n}^{0}$-formula $R\left(u, v_{1}, \ldots, v_{s}, \bar{b}\right)$ and suppose $a$ is an infinite integer of $\Re$ such that $\Re \vDash \operatorname{Cons}\left(T_{n}+\mathbb{Q}_{\bar{a}}\right)$. Then there is a $\Pi_{n}^{0}$-elementary end extension $\Re$ of $\Re$ such that $\Re=\mathbb{Q}_{n+2}^{0}$ and the standard integers are both $\exists^{n+1}$ and $\forall^{n+1}$ definable in $\mathcal{T}$ (in parameters $b$ and $a$ ).

PROOF. We define a function $f$ in $\pi$ such that for $r \leqslant a$ in the domain of $f$, we have $\Re \vDash \operatorname{Prov}\left[\mathbb{Q}_{\overline{f(r)}} ; \operatorname{Cons}\left(T_{n}+\mathbb{Q}_{\bar{r}}\right)\right]$. This can be done by setting

$q=f(r) \equiv q<\bar{a} \wedge r<\bar{a}$

$$
\begin{aligned}
\wedge \exists u(u<\bar{a} & \wedge(u)_{0}=q \wedge \operatorname{Prf}\left((u)_{1} ; \mathbb{Q}_{q} ; \overline{\operatorname{Cons}}\left(T_{n}+\tilde{\mathbb{Q}}_{r}\right)\right) \\
& \left.\wedge \forall v\left(v<u \rightarrow \neg \operatorname{Prf}\left((v)_{0} ; \mathbb{Q}_{(v)} ; \overline{\operatorname{Cons}}\left(T_{n}+\tilde{\mathbb{Q}}_{r}\right)\right)\right)\right) .
\end{aligned}
$$

(In paraphrase -" $f(r)$ is that $q<a$ such that $\mathbb{Q}_{q}$ proves $\operatorname{Cons}\left(T_{n}+\mathbb{Q}_{r}\right)$ with proof of shortest length".) This function $f$ is $\Delta_{n+1}^{0}$-definable in $\Re$ in the parameters $b$ and $a$. By Theorem 1.6, $f$ is defined on all standard $r$; hence, the domain of $f$ is an infinite integer $<a$. We also remark that the above definition of $f$ is absolute in all $\Pi_{n}^{0}$-elementary and extensions of $\Re$ which satisfy $\mathbb{Q}_{n+2}^{0}$. Let $m_{1}$ be a standard integer sufficiently large so that

$$
\Re \vDash \forall x\left(x>\bar{m}_{1} \wedge x \in \operatorname{dom} \bar{f} \rightarrow x<\bar{f}(x)\right) .
$$

(That such $m_{1}$ exists can be established using Lemma 3.1, for example.) We next define in $\Re$ a sequence $k_{i}$ of elements of the domain of $f$ by setting $k_{0}=m_{1}+1, k_{i+1}=f\left(k_{i}\right)$; let $c$ be the length of this sequence; so $c$ is an infinite integer of $\mathscr{N}$ and $c<a$. We now set $l_{s}=k_{c-s}$. The domain of the 
sequence $l_{s}$ is an infinite integer of $\mathscr{R}$; moreover, $\mathfrak{R}_{0}=\exists \psi\left(\operatorname{Tr}_{n}(\psi) \wedge\right.$ $\left.\operatorname{Prov}\left(\psi, \mathbb{Q}_{k} ; \overline{\operatorname{Cons}}\left(T_{n}+\tilde{\mathbb{Q}}_{l_{j+1}}\right)\right)\right)$ for $s \in \mathrm{N}$. So with $S=\left\{k: \Re_{0} \vDash \bar{k}<l_{s}\right.$, for all $s \in \mathrm{N}\}$, the sequence $l_{s}, s \in \mathrm{N}$, and $S$ satisfy the hypotheses of Theorem 3.3. Suppose, as in the proof of that theorem, that we have constructed end extensions $\Re_{0}<_{n} \Re_{1}<_{n} \cdots<_{n} \Re_{s}$ such that $\Re_{s} \vDash \operatorname{Cons}\left(T_{n}+\mathbb{Q}_{l_{r}}\right)$ and $\mathfrak{T}_{s} \models \neg \operatorname{Cons}\left(T_{n}+\mathbb{Q}_{l_{t}}\right)$ for all $t<s$, and also suppose $\mathfrak{T}_{s} \vDash$ $M_{n}\left(l_{t}, \bar{m}\right)$ for all $t<s$ and all standard $m$. Let $k \in\left|\Re_{s}\right|$ be an infinite integer; since $\mathfrak{N}_{s} \vDash \operatorname{Cons}\left(T_{n}+\mathbb{Q}_{l_{s}}\right)$, we have, by Friedman's lemma, that $\Re_{s}=\operatorname{Cons}\left(T_{n}+\mathbb{Q}_{l_{s}}+S_{n}\left(\tilde{l}_{s}, \tilde{k}\right)\right)$. Therefore we can take $\Re_{s+1}$ to satisfy $S_{n}(\bar{l}, \bar{k})$ obtaining

$$
\Re_{s+1} \vDash \mathbb{Q}+\operatorname{Cons}\left(T_{n}+\mathbb{Q}_{l_{s+1}}\right)+\neg \operatorname{Cons}\left(\mathbb{Q}_{\bar{l}}\right)+\neg M_{n}\left(\bar{l}_{s}, \bar{k}\right)
$$

and

$$
\Re_{s+1} \vDash M_{n}\left(\bar{l}_{s}, \bar{m}\right) \text { for all standard } m \text {. }
$$

We next claim that for $t \geqslant s+1$, we will have $\Re_{t} \vDash M_{n}\left(\bar{l}_{s}, \bar{m}\right)$ for all standard $m$ and also $\Re_{t} \models \neg M_{n}\left(\bar{l}_{s}, \bar{k}\right)$; this follows from the fact that $\Re_{s+1}$ $F \neg \operatorname{Cons}\left(\mathscr{Q}_{k_{5}}\right)$ and so for $m \in N$, there exist $x_{0}, \rho_{0}$ and $\phi_{0} \in|\Re|_{s+1}$ such that

$$
\begin{aligned}
\mathscr{N}_{s+1} & \vDash \operatorname{Tr}_{n}\left(\bar{\phi}_{0}\right) \wedge “ \bar{\rho}_{0} \text { is a disjunction of the form } \\
& \pm \mu_{0}^{\bar{l}} \vee \cdots \vee \pm \mu_{m}^{\frac{\bar{l}_{s}}{m}} \text { ” } \\
& \wedge \operatorname{Prf}\left(\bar{x}_{0} ; \bar{\phi}_{0}, \mathbb{Q}_{\bar{l}_{s}} ; \bar{\rho}_{0} \vee \neg \mu_{m}^{\bar{l}}\right) \\
& \wedge \forall y<\bar{x}_{0} \forall \psi<\bar{x}_{0}\left(\operatorname{Tr}_{n}(\psi) \rightarrow \neg \operatorname{Prf}\left(y ; \psi, \mathbb{Q}_{\bar{l}} ; \bar{\rho}_{0} \vee \mu \frac{\bar{l}_{m}}{m}\right)\right)
\end{aligned}
$$

and this is absolute in $\Pi_{n}^{0}$-elementary end extensions of $\Re_{s+1}$ which satisfy $\mathbb{Q}_{n+2}^{0}$; likewise, $\neg M_{n}\left(\bar{l}_{s}, \bar{k}\right)$ is $\Sigma_{n+1}^{0}$ and so persists in all such extensions of $\mathfrak{N}_{s+1}$.

Therefore we can construct the $\Pi_{n}^{0}$-elementary end extension chain $\Re_{s}, s$ $\in \mathbf{N}$, so that for all $s$,

(i) for every infinite $k \in\left|\Re_{s}\right|$, there is $t \geqslant s$ such that $\mathfrak{R}_{t+1} \vDash \mathbb{Q}+$ $\neg \operatorname{Cons}\left(T_{n}+\mathbb{Q}_{\bar{l}_{l}}\right)+\neg M_{n}\left(\bar{l}_{t}, \bar{k}\right)$;

(ii) for every standard $m, \mathfrak{N}_{s} \vDash M_{n}(\bar{l}, \bar{m})$.

Then the limit model $\mathfrak{\pi}_{\omega}=\cup_{s \in \mathrm{N}} \Re_{s}$ will satisfy

$$
k \in \mathbf{N} \Leftrightarrow \mathfrak{R}_{\omega} \vDash \neg \operatorname{Cons}\left(T_{n}+\mathbb{Q}_{l_{k}}\right)
$$

and

$$
k \in \mathbf{N} \Leftrightarrow \mathbb{R}_{\omega} \vDash \forall x\left(\neg \operatorname{Cons}\left(T_{n}+\mathscr{Q}_{l_{x}}\right) \rightarrow M_{n}\left(l_{x}, \bar{k}\right)\right) .
$$

Since $\neg \operatorname{Cons}\left(T_{n}+\mathbb{Q}_{l_{x}}\right)$ is $\Sigma_{n+1}^{0}$ (in $b$ and $a$ ) and since $M_{n}(x, y)$ is $\Pi_{n+1}^{0}$, the standard integers are thus $\Sigma_{n+1}^{0}$ and $\Pi_{n+1}^{0}$ definable (in $b$ and $a$ ) in $\mathfrak{T}_{\omega}$; 
because $\mathfrak{N}_{\omega} \models \mathscr{P}_{n+2}^{0}$, the standard integers are in fact $\exists^{n+1}$ and $\forall^{n+1}$ definable in $\Re_{\omega}$ (in $b$ and $a$ ).

If the parameters $b$ and $a$ of the above theorem are themselves $\Pi_{n}^{0}$ minimal in $\mathfrak{N}$, then they can be eliminated from the formulas defining $\mathbf{N}$ in $\mathfrak{K}_{\omega}$; we thus have

THEOREM 6.2. Suppose $\mathfrak{T}$ is a countable model of $\mathbb{Q}$ in which $\mathbb{A}$ is represented by a $\Pi_{n}^{0}$-formula $R\left(u, v_{1}, \ldots, v_{s}, \bar{b}\right)$ and suppose $k$ is an infinite integer of $\mathfrak{N}$ such that $\mathfrak{N} \vDash \operatorname{Cons}\left(T_{n}+A_{\bar{k}}\right)$. If $b$ and $k$ are $\Pi_{n}^{0}$-minimal in $\Re$, then there is a $\Pi_{n}^{0}$-elementary end extension $\Re$ of $\mathscr{N}$ such that $\Re \vDash \mathbb{Q}_{n+2}^{0}$ and the standard integers are $\exists^{n+1}$ - and $\forall^{n+1}$-definable without parameters in r.

Our next result which is based on work of Feferman, cf. [Fef], complements the above theorem.

LEMMA 6.3. Let $n$ and $m$ be nonnegative integers and let $\mathbb{Q}$ be represented in $\mathbf{N}$ by a $\Pi_{n}^{0}$-formula $R\left(u, v_{1}, \ldots, v_{s}\right)$. Suppose that $Q$ is consistent with $\mathfrak{T}_{n}^{0}$. Then there is a model $\mathcal{O}$ of $Q+\mathscr{T}_{n}^{0}$ which satisfies $\operatorname{Cons}\left(T_{m}+Q_{\bar{k}}\right)$ for some infinite $k \in|\Re|$ which is $\Pi_{n}^{0}$-minimal in $\Re$.

Proof. Let $F$ be a closed formula satisfying

$$
\begin{aligned}
\mathcal{Q} \vdash F \leftrightarrow \forall x \forall y \forall z \forall \phi \forall u\left[G(x, y, z) \wedge \operatorname{Tr}_{n}(\phi)\right. & \wedge \operatorname{Prf}(u ; y, \phi ; \bar{F}) \\
& \left.\rightarrow \operatorname{Cons}\left(T_{m}+\mathcal{Q}_{\max (z, \phi, u, y, x)}\right)\right]
\end{aligned}
$$

where $\exists z G(x, y, z)$ is a $\Sigma_{n+1}^{0}$ definition of the function $y=\mathbb{Q}_{x}$. We claim that $\mathbb{Q}+\mathfrak{T}_{n}^{0}+\neg F$ is consistent: if not, there exist a proof $p$, a conjunction $A$ of axioms of $Q$ and a $\forall^{n}$-sentence $E$ which is satisfied in $\mathbf{N}$ such that $p$ is a proof of $F$ from $A, E$; let $\mathscr{T}$ be a model of $\mathcal{Q}+\mathfrak{T}_{n}^{0}$, then by our hypothesis, $\Re \vDash F$ and $\Re \vDash \operatorname{Tr}_{n}(\bar{E})$ and $\Re \vDash \operatorname{Prf}(\bar{p} ; \bar{A}, \bar{E} ; \bar{F})$; we can also suppose that for some standard $i, j$ we have $\mathfrak{N} \vDash G(\bar{i}, \bar{A}, \bar{j})$; hence, $\Re \vDash$ $\neg \operatorname{Cons}\left(T_{m}+Q_{\max (\bar{p}, \bar{E}, \bar{i}, \bar{A}, \bar{j})}\right)$ which is absurd. So, with the claim established, let $\Re$ be a model of $Q+\mathscr{T}_{n}^{0}+\neg F$. Then in $\Re$ take $k$ to be the least integer satisfying

$$
\exists x, y, z, u, \phi<k\left(G(x, y, z) \wedge \operatorname{Tr}_{n}(\phi) \wedge \operatorname{Prf}(u ; y, \phi ; \bar{F})\right) .
$$

Corollary 6.4. Suppose that $Q$ is represented in $\mathbf{N}$ by a $\Pi_{n}^{0}$-formula $R\left(u, v_{1}, \ldots, v_{s}\right)$ and that $\mathbb{Q}$ is consistent with $\mathscr{T}_{n}^{0}$. Then there is a nonstandard model $\Re$ of $\mathbb{Q}_{n+2}^{0}+\mathscr{T}_{n}^{0}$ in which the standard integers are $\exists^{n+1}$ - and $\forall^{n+1}$. definable without parameters.

LEMMA 6.5. Suppose $\mathbb{Q}$ is represented in $\Re$ by a $\Pi_{n}^{0}$-formula $R\left(u, v_{1}, \ldots, v_{s}\right)$ where $\Re$ is a model of $\mathcal{Q}$ and suppose that $\mathfrak{N} \vDash \operatorname{Cons}_{R}\left(T_{n}\right.$ 
$+\mathbb{Q})$. Then there is a $\Pi_{n}^{0}$-elementary end extension $\mathfrak{N}$ of $\mathfrak{N}$ which is a model of $\mathcal{Q}$ and which contains a $\Pi_{n}^{0}$-minimal infinite integer $k$ such that $\mathscr{N}=$ $\operatorname{Cons}_{R}\left(T_{n}+Q_{\bar{k}}\right)$.

PROOF. If $\mathfrak{N}$ satisfies

$$
\exists \phi \exists u \exists v\left(\operatorname{Tr}_{n}(\phi) \wedge \operatorname{Prf}\left(u ; \phi, \mathbb{Q}_{v} ; \overline{\neg \operatorname{Cons}_{R}\left(T_{n}+Q\right)}\right)\right)
$$

we can take $\Re=\mathfrak{T}$ and $k$ to be the least integer satisfying

$$
\exists x, y, z, \phi, y<k\left(G(x, y, z) \wedge \operatorname{Tr}_{n}(\phi) \wedge \operatorname{Prf}\left(u ; \phi, y ; \overline{\neg \operatorname{Cons}_{R}\left(T_{n}+\mathbb{Q}\right)}\right)\right)
$$

where again $\exists z G(x, y, z)$ is a $\Sigma_{n+1}^{0}$ definition of $y=\mathbb{Q}_{x}$ in $\mathfrak{K}$.

If $\Re$ does not satisfy (*), then we can apply Theorem 3.2 to $\mathscr{N}$ and the theory $\mathbb{Q}+\operatorname{Cons}_{R}\left(T_{n}+\mathscr{Q}\right)$ to find an $n$-elementary end extension of $\mathfrak{R}$ which satisfies (*) and $Q+\operatorname{Cons}_{R}\left(T_{n}+Q\right)$.

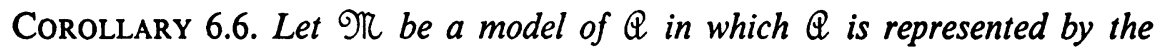
$\Pi_{n}^{0}$-formula $R\left(u, v_{1}, \ldots, v_{s}\right)$ and suppose that $\mathfrak{N} \vDash \operatorname{Cons}_{R}\left(T_{n}+\mathbb{Q}\right)$. Then $\mathfrak{N}$ has a $\Pi_{n}^{0}$-elementary end extension $\mathscr{N}$ such that $\mathscr{N} \vDash \mathbb{Q}_{n+2}^{0}$ and the standard integers are $\exists^{n+1}$ - and $\forall^{n+1}$-definable in $\Re$ without parameters.

REMARK 6.7. It follows from the above corollary that if $Q$ is recursively enumerable and if $\Re$ is a countable model of $\mathcal{Q}$ such that $\mathscr{N} \vDash \operatorname{Cons}_{R}(\mathbb{Q})$, for some $\Pi_{0}^{0}$-representation, $R\left(x, y_{1}, \ldots, y_{s}\right)$ of $Q$ in $\mathbf{N}$, then $\mathscr{N}$ has an end extension $\Re$ which is a model of $\mathbb{Q}_{2}^{0}$ and in which the standard integers are existentially definable without parameters. On the other hand, for recursively enumerable $Q$ it is not difficult to construct a countable nonstandard model $\mathfrak{T}$ of $\mathbb{Q}$ such that the standard integers are existentially definable without parameters in no extension of $\mathbb{R}$ which satisfies $\mathbb{Q}_{2}^{0}$-one simply takes $\mathscr{R}$ to be an extension of an existentially complete model of $\mathbb{Q}_{2}^{0}$.

A model of $\mathscr{P}_{2}^{0}$ which is the limit of an end extension chain of models of $\mathscr{P}$ is easily seen to be a model of Primitive Recursive Arithmetic. Since the model of Corollary 6.6 is obtained as the limit of such a chain, we have

THEOREM 6.8. There is a nonstandard model $\mathfrak{N}$ of Primitive Recursive Arithmetic in which the standard integers are $\exists^{1}$ - and $\forall^{1}$-definable without parameters. Therefore, parameter free $\Delta_{1}^{0}$-induction is not provable in Primitive Recursive Arithmetic.

We conclude this section with two corollaries to the proof of Theorem 6.1 and some remarks.

THEOREM 6.9. Let $\mathfrak{N}$ be an initially countable model of $\mathbb{Q}$ in which $\mathbb{Q}$ is represented by a $\Pi_{n}^{0}$-formula $R\left(u, v_{1}, \ldots, v_{s}, \bar{b}\right)$. Suppose that $a$ is an infinite integer of $\mathfrak{N}$ such that $\mathfrak{N} \vDash \operatorname{Cons}\left(T_{n}+\mathbb{Q}_{\bar{a}}\right)$. Then $\mathfrak{R}$ has a $\Pi_{n}^{0}$-elementary 
end extension which is a model of $\mathbb{Q}_{n+2}^{0}$ and in which $\mathbf{N}$ is $\Delta_{n+1}^{0}$-definable (from parameters $b$ and $a$ ).

THEOREM 6.10. Let $\mathfrak{N}$ be a model of $\mathbb{Q}$ in which $\mathbb{Q}$ is represented by $a$ $\Pi_{n}^{0}$-formula $R\left(u, v_{1}, \ldots, v_{s}, \bar{b}\right)$. Suppose that $a$ is an infinite integer of $\Re$ such that $\Re \vDash \operatorname{Cons}\left(T_{n}+\mathbb{Q}_{\bar{a}}\right)$. Then $\Re$ has a $\Pi_{n}^{0}$-elementary end extension which satisfies $\mathbb{Q}_{n+2}^{0}$ and in which $\mathbf{N}$ is $\exists^{n+1}$-definable (from parameters $b$ and $a$ ).

REMARK 6.11. We do not know whether the hypothesis that $\mathfrak{T}$ be initially countable is necessary in Theorem 6.10. Note that in Theorem 6.11, $\mathfrak{N}$ need not be assumed initially countable. Also, cf. Corollary 3.4 and Theorem 5.4.

REMARK 6.12. Since $\mathbf{N}$ cannot be $\Sigma_{1}^{0}$-definable in nonstandard existentially complete models of $\mathbb{A}_{2}^{0}$, the models constructed in the proof of Theorem 6.1 for the case $n=0$ are not existentially complete, cf. $\$ 8$ of $[\mathbf{H}, \mathbf{W}$; for a positive result showing that some $\Delta_{1}^{0}$-induction is provable in $\mathbb{Q}_{2}^{0}$, again see $\S 8$ of $[\mathbf{H}, \mathbf{W}]$.

7. Limitations of the truncated theories $\mathbb{Q}_{n}^{0}$. In $\S \S 3$ and 6, we constructed models of the truncated theories $\mathbb{Q}_{n+2}^{0}$ where instances of the induction scheme failed. We now give some further illustrations of weaknesses of these theories. Recall the following well-known positive results for $\mathcal{P}$ :

TheOREM A (MACDowell-SpeCKer Theorem). Every countable model of 9 has a proper elementary end extension.

Theorem B (Strong Reflection Scheme). For all formulas $F\left(x_{1}, \ldots, x_{n}\right)$, $\mathscr{P} \vdash \forall x_{1} \cdots \forall x_{n}\left(F\left(x_{1}, \ldots, x_{n}\right) \rightarrow \operatorname{Cons}\left(\bar{F}\left(\tilde{x}_{1}, \ldots, \tilde{x}_{n}\right)\right)\right)$.

Theorem C (Arithmetized Completeness Theorem at $n$ ). Fix $n \geqslant 0$. Let Th be a model of $\mathcal{P}$ and let $F$ be a sentence of the language of arithmetic such that $\Re \vDash \operatorname{Cons}\left(T_{n}+\bar{F}\right)$. Then there is $a \forall^{n}$-elementary end extension $\Re$ of IT which satisfies $F$.

Remark 7.1. For Theorem A, we refer the reader to [M, Sp], [K, M] or [G, bis]. Theorem $B$ is Scheme 1.5 together with Theorem 1.6. Theorem $C$ is Theorem 1.7.

We shall now show that Theorems A, B, C all fail for the truncated theories $\mathbb{Q}_{n+2}^{0}$.

THEOREM 7.2. Let $n \geqslant 0$. Let $\mathfrak{T}_{0}$ be a countable model of $\mathbb{Q}$ in which $\mathbb{Q}$ is represented by means of a $\Pi_{0}^{0}$-formula $R\left(u, v_{1}, \ldots, v_{s}, \bar{b}\right)$ and let $\Re_{\omega}$ be a $\Pi_{n}^{0}$-elementary end extension of $\Re_{0}$ such that $\Re_{0}$ and $\Re_{\omega}$ satisfy the conclusion of Theorem 5.6. Then

(a) $\Re_{\omega}$ has no proper $\forall^{n}$-elementary end extension which satisfies $\mathscr{P}_{n+2}^{0}$.

(b) There is a formula $F\left(u_{1}, \ldots, u_{t}\right)$ such that 


$$
\Re_{\omega} \vDash \exists u_{1}, \ldots, \exists u_{t}\left(F\left(u_{1}, \ldots, u_{t}\right) \wedge \neg \operatorname{Cons}\left(\bar{F}\left(\tilde{u}_{1}, \ldots, \tilde{u}_{t}\right)\right)\right) .
$$

(c) There is a theorem $A$ of $\mathbb{Q}$ such that $\mathfrak{M}_{\omega} \vDash \operatorname{Cons}\left(T_{n}+\bar{A}\right)$, but there is no $\forall^{n}$-elementary end extension of $\mathfrak{R}_{\omega}$ which satisfies $A$.

Proof. (a) The nonstandard integers are $\Sigma_{n+1}^{0}$ definable in $\mathfrak{N}_{\omega}$ as $\{x$ : $\left.\neg \operatorname{Cons}\left(T_{n}+\mathbb{Q}_{x}\right)\right\}$. Let $H(x, y, \bar{b})$ be a $\Pi_{n}^{0}$-formula so that $\neg \operatorname{Cons}\left(T_{n}+\mathbb{Q}_{x}\right) \equiv$ $\exists y H(x, y, \bar{b})$. Suppose $\Re$ is a proper $\forall^{n}$-elementary end extension of $\Re_{\omega}$ which satisfies $\mathscr{P}_{n+2}^{0}$; then $\Re$ is a $\Pi_{n}^{0}$-elementary extension of $\Re_{\omega}$. Also, for standard $m$, we have $\Re \vDash \operatorname{Cons}\left(T_{n}+Q_{\bar{m}}\right)$ by Theorem 1.6. So let $c \in|\Re|$ - |ฯ|. Now consider $K(\bar{c}, x, \bar{b}) \equiv \exists y<\bar{c} H(x, y, \bar{b})$. The formula $K(\bar{c}, x, \bar{b})$ defines a set in $\Re$ with no least element; since $K(z, x, w)$ is equivalent in $\mathscr{P}_{n+2}^{0}$ to a $\Pi_{n}^{0}$-formula, this violates an instance of the induction scheme which is provable in $\mathscr{P}_{n+2}^{0}$.

(b) Let $\mathcal{Q}$ denote R. M. Robinson's finitely axiomatizable subtheory of $\mathscr{P}$ all of whose models are end extensions of the standard integers; and let $Q \equiv M 2$. Let $a$ be an infinite integer of $\mathfrak{T R}_{0}$. Set

$$
\begin{aligned}
I(\bar{a}, \bar{b}) \equiv\left[\left(\operatorname{Cons}\left(T_{n}+\mathbb{Q}_{\overline{0}}\right) \wedge \forall x((x<\bar{a}\right.\right. & \left.\wedge \operatorname{Cons}\left(T_{n}+\mathbb{Q}_{x}\right)\right) \\
& \left.\left.\rightarrow \operatorname{Cons}\left(T_{n}+\mathbb{Q}_{x+1}\right)\right)\right) \\
& \left.\rightarrow \forall x\left(x<\bar{a} \rightarrow \operatorname{Cons}\left(T_{n}+\mathbb{Q}_{x}\right)\right)\right] .
\end{aligned}
$$

LEMMA 7.3. Consider the parameters $\bar{a}$ and $\bar{b}$ of the formulas $\operatorname{Cons}\left(T_{n}+\mathbb{Q}_{x}\right)$ and $I(\bar{a}, \bar{b})$ as variables. We have

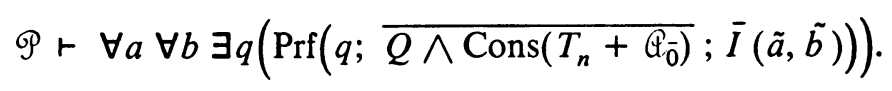

Proof of LEMMA. Let $\mathscr{T} \vDash \mathscr{P}$, let $a, b \in|\mathscr{T}|$ and set $K \equiv Q \wedge \operatorname{Cons}\left(T_{n}\right.$ $\left.+Q_{\overline{0}}\right) \wedge \neg I(\bar{a}, \bar{b})$. Suppose that $K$ is consistent in $\mathscr{R}$. By the Arithmetic Completeness Theorem, there is a model $\Re$ of $K$ which is arithmetically definable in $\Re$. By the property of 2 mentioned above, this model $\Re$ is necessarily an end extension of $\Re$. So $a, b \in|\Re|$. Therefore $X=\{x: \Re \vDash$ $\left.\bar{x}<\bar{a} \wedge \neg \operatorname{Cons}\left(T_{n}+Q_{x}\right)\right\}$ is a nonempty finite set in $\Re$ of integers $<a$. Also $0 \notin X$ since $\Re \vDash \operatorname{Cons}\left(T_{n}+\mathbb{Q}_{\overline{0}}\right)$. By induction in $\Re, X$ has a least element $k_{0}$ with $0<k_{0}<a$. So $\Re \vDash \operatorname{Cons}\left(T_{n}+Q_{\overline{k_{0}-1}}\right) \wedge \overline{\left(k_{0}-1\right)}<\bar{a}$; as $\Re \vDash \neg I(\bar{a}, \bar{b})$, we have $\Re \vDash \operatorname{Cons}\left(T_{n}+\mathbb{Q}_{\bar{k}_{0}}\right)$, which contradicts the choice of $k_{0}$. Since $\Re$ was arbitrary, the lemma is proved.

Since the lemma shows that a certain $\Pi_{2}^{0}$ sentence is a theorem of $\mathscr{P}$, we obtain

$$
\mathfrak{R}_{\omega} \vDash \operatorname{Prov}\left[Q \wedge \operatorname{Cons}\left(T_{n}+\mathbb{Q}_{\overline{0}}\right) ; I(\bar{a}, \bar{b})\right] .
$$

But $\Re_{\omega} \vDash Q \wedge \operatorname{Cons}\left(T_{n}+Q_{\overline{0}}\right) \wedge \neg I(\bar{a}, \bar{b})$, so with $H(\bar{a}, \bar{b}) \equiv Q \wedge$ 
$\operatorname{Cons}\left(T_{n}+\mathbb{Q}_{\overline{0}}\right) \wedge \neg I(\bar{a}, \bar{b})$, we have

$$
\Re_{\omega} \vDash \exists u, v(H(u, v) \wedge \neg \operatorname{Cons}(\bar{H}(\tilde{u}, \tilde{v}))) .
$$

(c) In [M, S], Macintyre and Simmons generalize results of Hirschfeld and construct a formula $S^{(n)}(x)$ of type $\exists^{n+1}$ such that, setting $I(x) \equiv \exists y(x<y$ $\left.<2 x \wedge \neg S^{(n)}(x)\right)$, one obtains

(i) $\mathcal{P} \vdash \forall x I(x)$.

(ii) For all $\Re \in E_{n}(\mathbb{Q})$, for all nonstandard $k \in|\Re|$, $\Re \vDash \neg I(\bar{k})$.

(The set $S^{(0)}(x)$ is Post's simple set, cf. $\S 8$ of [Ro], and $\S 8$ of [H, W], and the $S^{(n)}(x), n \geqslant 1$, are variants of it.)

Note that, since $\Re_{\omega} \in E_{n}(\mathbb{Q})$, for infinite $k \in|\mathscr{R}|$ and all $\forall^{n}$-elementary end extensions $\Re$ of $\Re$, we have $\Re \vDash S^{(n)}(\bar{k})$. The sentence $\operatorname{Cons}\left(T_{n}+\overline{\forall x I(x)}\right)$ is a $\Pi_{n+1}^{0}$ theorem of $\mathcal{P}$ by (i) and Theorem 1.6. Hence $\mathfrak{R}_{\omega} \vDash \operatorname{Cons}\left(T_{n}+\forall x I(x)\right)$; however, no $\forall^{n}$-elementary end extension of $\mathfrak{T}_{\omega}$ satisfies $\forall x I(x)$.

Consider the following Weak Reflection Scheme:

$F \rightarrow \operatorname{Cons}(\bar{F})$, all closed formulas $F$.

This scheme is provable in $\mathscr{T}_{2}^{0}$ : for if $\mathfrak{T} \vDash \mathfrak{T}_{2}^{0}+F$, then $F$ is consistent, so $\operatorname{Cons}(\bar{F})$ is a $\Pi_{1}^{0}$-sentence which holds in $\mathbf{N}$; therefore $\mathscr{T}_{2}^{0} \vdash \operatorname{Cons}(\bar{F})$. However, the Weak Reflection Scheme is not provable in $\mathbb{Q}_{n+2}^{0}$ for any $n$, if $\mathbb{Q}$ is recursively enumerable as the following theorem shows:

THEOREM 7.4. Let $\mathbb{Q}$ be a recursively enumerable extension of $\mathcal{P}$. Then the Weak Reflection Scheme is not provable in $\mathbb{Q}_{n}^{0}$, for any $n$.

Proof. It suffices to eliminate the parameters $\bar{a}, \bar{b}$ in the proof of part (b) of Theorem 7.2. As $\mathbb{Q}$ is recursively enumerable, the parameter $\bar{b}$ disappears and for $\bar{a}$ we can take a $\Pi_{0}^{0}$-minimal nonstandard element of $\Re_{0}$-we can assume such elements exist by virtue of Theorem 4.4.

Finally, looking more closely at the proof of Theorem 7.2(c), we note that in the case $n=0$, the class $T_{n}$ can be replaced by the finitely axiomatizable theory 2. We then obtain as a corollary to Theorem 7.2(c)

THEOREM 7.5. There is a model $\Re$ of $\mathbb{Q}_{2}^{0}$ and a theorem $A$ of $\mathcal{P}$, such that $\Re$ $\vDash \operatorname{Cons}(\overline{Q \wedge A})$ but no end extension of $\Re$ satisfies $Q \wedge A$.

Remarks 7.6. This last result shows that the Henkin argument to prove the Completeness Theorem cannot be formalized in $\mathfrak{T}_{2}^{0}$ even for single standard formulas. It also shows that the proof of the Gödel Completeness Theorem cannot be formalized in $\mathfrak{T}_{2}^{0}$. These arguments can however be carried out in $\mathscr{P}_{3}^{0}$. 


\section{BIBLIOGRAPHY}

[B] J. Barwise, Infinitary methods in the model theory of set theory, Logic Colloquium '69, North-Holland, Amsterdam, 1971, pp. 53-66. MR 43 \#3103.

[Fef] S. Feferman, Arithmetization of metamathematics in a general setting, Fund. Math. 49 (1960/61), 35-92. MR 26 \#4913.

[Fr] H. Friedman, Some applications of Kleene's methods for intuitionistic systems, Cambridge Summer School in Mathematical Logic, Lecture Notes in Math., vol. 337, Springer-Verlag, Berlin and New York, 1973, pp. 113-170. MR 51 \# 12486.

[G] H. Gaifman, $A$ note on models and submodels of arithmetic, Conf. in Math. Logic - London '70, Lecture Notes in Math., vol. 255, Springer-Verlag, Berlin and New York, 1972, pp. 128-144.

[G, bis] __ Uniform extension operators for models and their applications, Sets, Models and Recursion Theory, North-Holland, Amsterdam, 1967, pp. 122-155. MR 36 \#3640.

[G, K, T] R. Gandy, G. Kreisel and W. Tait, Set existence, Bull. Acad. Polon. Sci. Sér. Sci. Math. Astronom. Phys. 8 (1960), 577-582. MR 28 \#2964a.

[G, M, S] D. C. Goldrei, A. Macintyre and H. Simmons, The forcing companions of number theories, Israel J. Math. 14 (1973), 317-337. MR 48 \# 5853.

[H, B] D. Hilbert and P. Bernays, Grundlagen der Mathematik. II, Springer, Berlin, 1939.

[H, W] J. Hirschfeld and W. Wheeler, Forcing, arithmetic, division rings, Lecture Notes in Math., vol. 454, Springer-Verlag, Berlin and New York, 1975. MR 52 \# 10412.

[K, L] G. Kreisel and A. Lévy, Reflection principles and their use for establishing the complexity of axiomatic systems, Z. Math. Logik Grundlagen Math. 14 (1968), 97-142. MR 37 \#3914.

$[\mathrm{K}, \mathbf{M}]$ J. L. Krivine and K. McAloon, Forcing and generalized quantifiers, Ann. Math. Logic 5 (1973), 199-255.

[K, M, bis] _ Some true unprovable formulas for set theory, Proc. Bertrand Russell Memorial Logic Conf., Leeds, 1973, pp. 332-341. MR 50 \# 9580.

[K, T] G. Kreisel and G. Takeuti, Formally self-referential propositions for cut free classical analysis and related systems, Dissertations Math. (Rozprawy Mat.) 118 (1974), 55 pp. MR 52 \#5374.

[K, W] G. Kreisel and H. Wang, Some applications of formalized consistency proofs, Fund. Math. 42 (1955), 101-110. MR 17, 447.

[Man] L. Manewitz, Internal end-extensions of Peano arithmetic and a problem of Gaifman, J. London Math. Soc. 13 (1976), 80-82.

[Ma] Ju. V. Matijasevič, Eunumerable sets are diophantine, Dokl. Akad. Nauk SSSR 191 (1970), 279-282 = Soviet Math. Dokl. 11 (1970), 354-358. MR 41 \#3390.

[Mc] K. McAloon, Applications alternées de théorèmes d' incomplétude et de théorèmes de complétude, C. R. Acad. Sci. Paris Sér. A-B 280 (1975), no. 13, Ai, A849-A852. MR 51 \#5295.

[Mc, bis] _ Formules de Rosser pour ZF, C. R. Acad. Sci. Paris Sér. A-B 281 (1975), no. 16, Ai, A669-A672. MR 52 \#5417.

[Mc, ter] _ Consistency statements and number theories, Proc. 1975 Logic Colloq. at Clermont-Ferrand (M. Guillaume, Editor), Publ. C.N.R.S., 1977.

[M, Sp] R. Mac Dowell and E. Specker, Modelle der Arithmetik, Infinitistic Methods, Pergamon, Oxford; PWN, Warsaw, 1961, pp. 257-263. MR 27 \#2425.

[M, S] A. Macintyre and H. Simmons, Algebraic properties of number theories, Israel J. Math. 22 (1975), 7-27.

[Mon] R. Montague, Semantical closure and non-finite axiomatizability. I, Infinitistic Methods, Pergamon, Oxford; PWN, Warsaw, 1961, pp. 45-69. MR 27 \# 38.

[Mos] A. Mostowski, On models of axiomatic systems, Fund. Math. 39 (1952), 133-158. (1953) MR 14, 938.

[Mos, bis] __ A generalization of the incompleteness theorem, Fund. Math. 49 (1960/61), 205-232. MR 24 \# A41.

[Ra] M. Rabin, Non-standard models and independence of the induction axiom, Essays on the foundations of mathematics, Magnes Press, Hebrew Univ., Jerusalem, 1961, pp. 287-299. MR 28 \#4999. 
[Rog] H. Rogers, Jr., Theory of recursive functions and effective computability, McGraw-Hill, New York, 1967. MR 37 \#61.

[Ros] J. B. Rosser, Extensions of some theorems of Gödel and Church, J. Symbolic Logic 1 (1936), 89-91.

[Ry] C. Ryll-Nardzewski, The role of the axiom of induction in elementary arithmetic, Fund. Math. 39 (1952), 239-263. (1953) MR 14, 938.

[Sch] J. R. Shoenfield, Mathematical logic, Addison-Wesley, Reading, Mass., 1967. MR 37 \#1224.

[Sco] D. Scott, On constructing models for arithmetic, Infinitistic Methods, Pergamon, Oxford; PWN, Warsaw, 1961, pp. 235-255. MR 27 \#2423.

[Sim] H. Simmons, Existentially closed structures, J. Symbolic Logic 37 (1972), 293-310. MR 51 \#12518.

[Sm] C. Smorynski, Applications of Kripke models, Metamathematical Investigation of Intuitionistic Arithmetic and Analysis, Lecture Notes in Math., vol. 344, Springer-Verlag, Berlin and New York, 1973.

[Sm, bis] __ Consistency and related metamathematical properties, Report 75-02, Dept. of Math., Univ. of Amsterdam, 1975.

[S, W] Y. Suzuki and G. Wilmers, Non-standard models for set theory, Proc. Bertrand Russell Memorial Logic Conf., Leeds, 1973, pp. 278-314. MR 50 \# 4302.

[W] A. Wilkie, On models of arithmetic-Answers to two questions raised by H. Gaifman, J. Symbolic Logic 40 (1973), 41-47.

[Wi] G. Wilmers, Thesis, Manchester, 1975.

UeR of Mathematics, University of Paris Vil, Tour 45-55-5me Etage, 2 Place Jussieu, 75221 Paris, France 